\title{
The solar wind at solar maximum: comparisons of EISCAT IPS and in situ observations
}

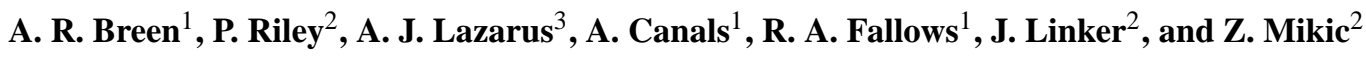 \\ ${ }^{1}$ University of Wales Aberystwyth, Penglais, Aberystwyth, Ceredigion, Wales, UK \\ ${ }^{2}$ Science Applications International Corporation, La Jolla, California, USA \\ ${ }^{3}$ Massachusetts Institute for Technology, Cambridge, Massachusetts, USA
}

Received: 12 November 2001 - Revised: 12 July 2002 - Accepted: 19 July 2002

\begin{abstract}
The solar maximum solar wind is highly structured in latitude, longitude and in time. Coronal measurements show a very high degree of variability, with large variations that are less apparent within in situ spacecraft measurements. Interplanetary scintillation (IPS) observations from EISCAT, covering distances from 20 to 100 solar radii $\left(R_{S}\right)$, are an ideal source of information on the inner solar wind and can be used, therefore, to cast light on its evolution with distance from the Sun. Earlier comparisons of in situ and IPS measurements under solar minimum conditions showed good large-scale agreement, particularly in the fast wind. In this study we attempt a quantitative comparison of measurements made over solar maximum by EISCAT $\left(20-100 R_{S}\right)$ and the Wind and Ulysses spacecraft (at $215 R_{S}$ and $300-1000 R_{S}$, respectively). The intervals studied were August-September 1999, May 2000, September 2000 and May 2001, the last-named being the period of the second Ulysses fast latitude scan. Both ballistic and - when possible - MHD/ballistic hybrid models were used to relate the data sets, and we compare the results obtained from these two mapping methods.

The results of this study suggest that solar wind velocities measured in situ were less variable than those estimated from IPS measurements closer to the Sun, with the greatest divergence between IPS velocities and in situ measurements occurring in regions where steep longitudinal velocity gradients were seen in situ. We suggest that the interaction between streams of solar wind with different velocities leads to "smoothing" of solar wind velocities between 30-60 $R_{S}$ and $1 \mathrm{AU}$, and that this process continues at greater distances from the Sun.
\end{abstract}

Key words. Interplanetary physics (solar wind plasma; sources of the solar wind; instruments and techniques)

\section{Introduction}

In situ measurements from plasma instruments on spacecraft provide absolute measurements of solar wind parameters at

Correspondence to: A. R. Breen (azb@aber.ac.uk) a known position and time with very good time resolution, but as these are normally single-point measurements it is difficult to separate variations in space and time. Some multispacecraft experiments have been carried out (e.g. Rhodes and Smith, 1981; Miyake et al., 1988; Gazis, 1995; Bothmer and Schwenn, 1998), and these have provided considerable information on solar wind structure in interplanetary space. More serious, however, is the limited coverage of the solar wind outside the plane of the ecliptic and close to the Sun. Therefore, there are no in situ measurements of the regions where the solar wind originates and where its largescale structure is determined.

Remote sensing observations are - at present - the only sources of information on the corona and inner solar wind. Compared to in situ observations, they have good spatial coverage, being able to observe the solar wind at any latitude, but the solar wind parameters must be derived from other measurements instead of being measured directly, and the measurements themselves are normally path-integrated, containing contributions from different regions of solar wind. Remote sensing observations used to estimate solar wind speed include:

- Measurements of Doppler dimming (Hyder and Lites, 1970), in which relative intensities of spectral lines observed in UV are used to derive the wind speed;

- Measurements in which the drift speeds of bright features in white-light images that are assumed to be drifting in the background wind speed (e.g. Sheeley et al., 1997; Tappin et al., 1999).

Both of these methods provide extremely useful information on the corona and the innermost regions of the solar wind, but at present Doppler-dimming measurements are restricted to heliocentric distances inside $6 R_{S}$ (Kohl et al., 1995 ) and white-light measurements of drift speeds to inside $30 R_{S}$ (Brueckner et al., 1995). Measurements of interplanetary scintillation (IPS) can be used to extend coverage to greater heliocentric distances.

The rapid variation (scintillation) of the intensity of distant, small-diameter radio sources was first observed in the early 1960s. It was soon realised that measurements of this 


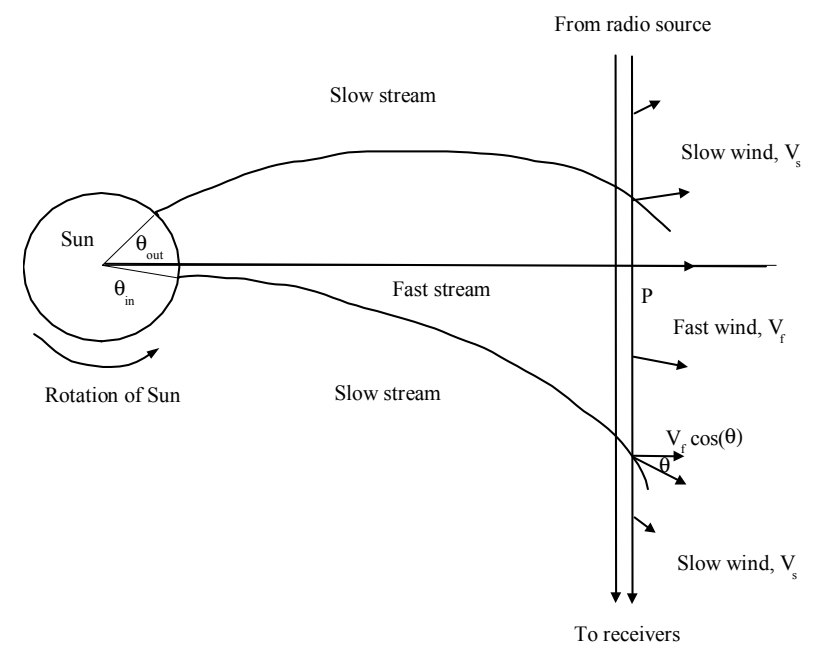

Fig. 1. Schematic diagrams of an IPS observation (not to scale): (a) Shown as if looking down on the North Pole of the Sun. The ray paths from the radio source to the receiver pass through the extended solar atmosphere, with their point of closest approach to the Sun (P) lying at a distance of $R_{S}$ solar radii from the centre of the Sun. $\theta_{\text {in }}$ and $\theta_{\text {out }}$ define the angular extent of the ray path which is immersed in fast solar wind: in the coordinate system adopted in this study, angles are measured from the Sun-P direction and are positive towards the radio source, and negative towards the Earth. Note that the fast stream does not need to be centred on the P-point - for example, in the observation of 1150-003 on 11 September 2000, mapping the IPS ray path down to $2.5 R_{S}$ and overlaying it on a map of whitelight intensity measured by LASCO C 2 suggested that $\theta_{\text {in }}=-80$ and $\theta_{\text {out }}=-20$.

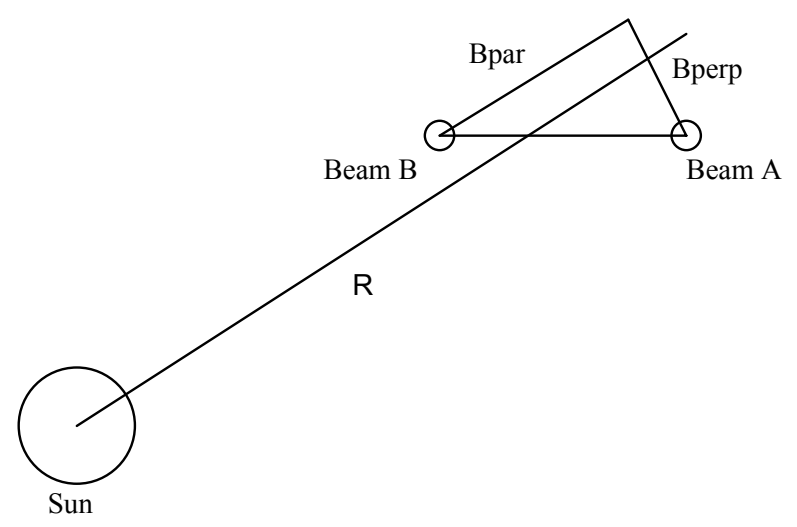

Fig. 1. (b) Shown as if looking from the direction of the radio source towards the receiving antennas. Beams A and B represent the ray paths from the source to the two receiving stations. The ray paths are separated by a distance $B_{\|}$is the direction radial to the Sun (shown as Bpar in the diagram) and by $B_{\perp}$ in the direction tangential to the Sun (Bperp in the diagram). The correlation between the scintillations seen at the two sites is greatest when $B_{\perp}$ is small. Velocity resolution increases for larger $B_{\|}$.

interplanetary scintillation (IPS) could provide information on the solar wind (e.g. Hewish et al., 1964; Dennison and Hewish, 1967). Developments in the technique led to the use of two-station observations, in which the scintillation pattern is measured at two widely separated sites (Armstrong and Coles, 1972). Provided that the IPS observations are made at a time when the ray paths from the radio source to the two receiving sites lie in a plane which passes through the centre of the Sun (Figs. 1a, b), a high degree of correlation may be present between the scintillation patterns seen at the two sites. To a first approximation the time-lag for maximum cross-correlation provides a direct estimate of the solar wind outflow speed (e.g. Bourgois et al., 1985), making it possible to obtain reliable measurements of the solar wind speed over a wide range of distances from the Sun and at any heliographic latitude (e.g. Kojima and Kakinuma, 1990; Coles, 1995). The accuracy to which the outflow speed can be determined improves as the baseline between the sites increases (e.g. Breen et al., 1996), as does the ability to resolve fast and slow streams when both are present in the scattering region (Rao et al., 1995).

IPS observations contain contributions to the scintillation pattern from the whole ray path, from the source to the receivers, but the scintillation potential (the scintillation power produced per unit length of the ray path through the solar wind) is determined by the variations in refractive index, which, in turn, are roughly proportional to the square of the electron density. In a uniform, radially expanding solar wind, the electron density falls off at a rate close to $R^{-2}$, so the scintillation potential drops off roughly as $R^{-4}$ (see Fallows, 2001; Fallows et al., 2002b for a more detailed treatment of scintillation levels), so the observation is normally dominated by scattering from the portion of the ray path nearest to the point of closest approach to the Sun (the P-point, as shown in Fig. 1a). The accuracy to which IPS can estimate solar wind speed improves as the separation of the antennas projected into the plane of the sky (the parallel baseline, $B_{\|}$, as illustrated in Fig. 1b) used to sample the scintillation pattern increases, with the time-lag for maximum correlation increasing, and as this happens, the ability to resolve two different solar wind speeds across the ray path improves (e.g. Grall, 1995; Rao et al., 1995; Grall et al., 1996).

In this study we use data from the EISCAT facility which offers $B_{\|}$up to $390 \mathrm{~km}$. EISCAT consists of three sites, at Ramfjordmoen (near Troms $\varnothing$, in Norway), Kiruna (Sweden) and Sodankylä (Finland), receiving over a $5 \mathrm{MHz}$ bandwidth centred on $931.5 \mathrm{MHz}$ (928.5 MHz in 2001). The EISCAT system was constructed as an ionospheric radar, and in this role is described by Rishbeth and Williams (1985), but its low-noise receivers, high timing accuracy and long baselines between sites make it a very powerful facility for IPS observations, as described by Bourgois et al. (1985) and Breen et al. $(1996,1998)$. The first IPS observations at EISCAT were made in 1982 (Bourgois et al., 1985) and regular, comprehensive campaigns have taken place every year during the arctic summer since 1990 (e.g. Coles et al., 1991). EISCAT observations of the low-density fast wind are clearly in the weak scattering regime outside $18-22 R_{S}$, while in the higher-density slow wind weak scattering can normally be assumed inside 25-30 $R_{S}$ (Fallows, 2001; Fallows et al., 
Table 1. EISCAT observations taken during the $3^{\text {rd }}$ Whole Sun Month, 13 August $1999-21$ September 1999 at latitudes within $\pm 15^{\circ}$ of the latitude of Wind or Ulysses. Wind lay at a mean heliographic latitude of $7^{\circ}$ during this period, while Ulysses climbed from $-32^{\circ}$ to $-34^{\circ}$ in the course of the month.

Latitudes and longitudes are in heliographic/Carrington coordinates projected ballistically back to $30 R_{S}$ at constant speed $V_{P}$, distances are heliocentric in units of solar radii $\left(R_{S}\right)$ and speeds are in $\mathrm{km} / \mathrm{s} . \theta_{\text {in }}$ and $\theta_{\text {out }}$ describe the position and angular extent of the fast stream, measured from the Sun-Earth line with angles +ve away from the Earth, as shown in Fig. 1 (if no angle shown, then the observation was fitted as a single stream). $V_{P}$ is the speed of the solar wind stream across the ray path at the point of closest approach to the Sun (the P-point) and, $d V_{\|}$is the variation in speed over the width of the stream (fast or slow) where this parameter was fitted, or the uncertainty in the fitted value of the stream speed (marked ${ }^{*}$ in the latter case). Observations shown in italics may be affected by strong scattering. The observation of $1042+120$ on 20 September 1999 may be a slow-rising CME. Source names are based on their Right Ascension and Declination in 2000 , with names given in the form hour, minute of right ascension + degrees of declination. The source $1008+075$, therefore, had a right ascension of $10 \mathrm{~h} 8 \mathrm{~min}$ and a declination of $+7.5^{\circ}$ at the start of 2000

\begin{tabular}{|c|c|c|c|c|c|c|c|c|c|}
\hline Date & Source & $\begin{array}{c}\text { Latitude } \\
\text { of P-point } \\
\text { (projected to } 30 R_{S} \text { ) }\end{array}$ & $\begin{array}{c}\text { Longitude } \\
\text { of P-point } \\
\text { (projected to } 30 R_{S} \text { ) }\end{array}$ & Limb & $\begin{array}{c}\text { Distance of } \\
\text { P-point }\end{array}$ & $\theta_{\text {in }}$ & $\theta_{\text {out }}$ & $V_{P}$ & $d V_{\|}$ \\
\hline 990813 & $1008+075$ & -20 & 349 & $\mathrm{E}$ & 43 & -50 & 15 & 555 & $15^{*}$ \\
\hline 990814 & $1008+075$ & -22 & 336 & E 40 & -65 & -30 & 360 & 202 & \\
\hline 990815 & $1008+075$ & -24 & 321 & $\mathrm{E}$ & 37 & -60 & -30 & 325 & 150 \\
\hline 990817 & $0854+201$ & +15 & 94 & W & 52 & - & - & 288 & 190 \\
\hline 990817 & $1008+075$ & -29 & 292 & $E$ & 30 & -80 & -45 & 317 & 102 \\
\hline 990818 & $1008+075$ & -32 & 277 & $E$ & 27 & - & - & 233 & 125 \\
\hline 990823 & $1058+015$ & -17 & 255 & $\mathrm{E}$ & 60 & -55 & -10 & 255 & 80 \\
\hline 990828 & $1229+020$ & +10 & 193 & $\mathrm{E}$ & 113 & - & - & 347 & 91 \\
\hline 990831 & $1008+075$ & -28 & 259 & W & 28 & - & - & 256 & 100 \\
\hline 990901 & $1229+020$ & +12 & 131 & $\mathrm{E}$ & 100 & - & - & 370 & 131 \\
\hline 990903 & $1008+075$ & -20 & 223 & W & 38 & - & - & 321 & 96 \\
\hline 990906 & $1229+020$ & +14 & 51 & $\mathrm{E}$ & 84 & - & - & 480 & 5 \\
\hline 990907 & $1229+020$ & +14 & 34 & $\mathrm{E}$ & 81 & - & - & 588 & 144 \\
\hline 990908 & $1229+020$ & +15 & 23 & $\mathrm{E}$ & 77 & - & - & 423 & 103 \\
\hline 990914 & $1008+075$ & -8 & 80 & W & 74 & -10 & 55 & 492 & 48 \\
\hline 990915 & $1008+075$ & -8 & 71 & W & 77 & - & - & 360 & 106 \\
\hline 990915 & $1042+120$ & +15 & 72 & W & 55 & - & - & 490 & 80 \\
\hline 990919 & $1008+075$ & -6 & 12 & W & 90 & -35 & 15 & 570 & 120 \\
\hline 990920 & $1008+075$ & -6 & 354 & W & 94 & -10 & 25 & 850 & 110 \\
\hline 990920 & $1042+120$ & +11 & 15 & W & 72 & - & - & 238 & 159 \\
\hline \multirow[t]{2}{*}{990921} & $1008+075$ & -5 & 344 & W & 97 & 0 & 50 & 620 & 145 \\
\hline & & & 1 & & & & & 259 & 32 \\
\hline
\end{tabular}

2002b). Reliable bulk speeds can be determined beyond $100 R_{S}$ for stronger radio sources.

Analysis of IPS data using a two-dimensional weak scattering model (Grall, 1995; Coles, 1996; Klinglesmith, 1997; Massey, 1998) have shown that, provided the observation is sufficiently far from the Sun as to be in weak scattering, it is possible to detect the presence of separate fast and slow streams across the ray path (Grall et al., 1996; Coles, 1996; Breen et al., 1996). In situ measurements have established that near to solar minimum, the solar wind has two distinct components: a fast stream flowing at about 750 to $800 \mathrm{~km} / \mathrm{s}$ and a slow stream with a flow speed of about 350 to $400 \mathrm{~km} / \mathrm{s}$ (e.g. Schwenn, 1990; Phillips et al., 1994; Woch et al., 1997; McComas et al., 2000). The fast wind originates from open magnetic field regions that are conspicuous as dark regions in maps of coronal white-light intensity (e.g. Snyder and Neugebauer, 1966; Krieger and Timothy, 1973; Neupert and Pizzo, 1974; Nolte et al., 1976). This bimodal distribution of solar wind speed has been confirmed in IPS data (e.g. Breen et al., 1996), and, together with the clear association between large regions of dark corona (coronal holes) and high-speed streams, makes it possible to use white-light intensity in coronal maps to determine whether a given region of an IPS ray path is immersed in fast or slow flow and thus, in the weak scattering regime, to separate the contributions of the observed scattering pattern of the fast and slow winds (e.g. Grall et al., 1996; Coles, 1996; Breen et al., 1998).

At solar maximum the situation is not so simple, and both in situ and IPS observations (Kojima and Kakinuma, 1990; Rickett and Coles, 1991; Fallows et al., 2002a) suggest a more continuous distribution of solar wind speeds. Nonetheless, coronal holes continue to be seen over solar maximum and regions of solar wind where enhanced flow speeds do still appear to be associated with these regions (e.g. Breen et 
Table 2. As Table 1 but summarizing observations taken by EISCAT between 11 and 28 May 2000 at latitudes within $\pm 15^{\circ}$ of the latitude of Wind or Ulysses. Wind lay at a mean heliographic latitude of $-2^{\circ}$ during this period, while Ulysses climbed from $-55^{\circ}$ to $-57^{\circ}$ in the course of the month. $V_{P}$ is the speed of the solar wind stream across the ray path at the point of closest approach to the Sun (the P-point) and, $d V_{\|}$is the variation in speed over the width of the stream (fast or slow) where this parameter was fitted, or the uncertainty in the fitted value of the stream speed (marked * in the latter case). Observations shown in italics may be affected by strong scattering

\begin{tabular}{|c|c|c|c|c|c|c|c|c|c|}
\hline Date & Source & $\begin{array}{c}\text { Latitude } \\
\text { of P-point } \\
\text { (projected to } 30 R_{S} \text { ) }\end{array}$ & $\begin{array}{c}\text { Longitude } \\
\text { of P-point } \\
\text { (projected to } 30 R_{S} \text { ) }\end{array}$ & Limb & Distance of & $\begin{array}{c}\theta_{\text {in }} \\
\text { P-point }\end{array}$ & $\theta_{\text {out }}$ & $V_{P}$ & $d V_{\|}$ \\
\hline 000511 & $0431+206$ & -11 & 11 & $\mathrm{E}$ & 67 & - & - & 431 & 200 \\
\hline 000512 & $0431+206$ & -11 & 359 & $\mathrm{E}$ & 63 & - & - & 320 & 120 \\
\hline 000514 & $0431+206$ & -12 & 327 & $\mathrm{E}$ & 57 & - & - & 369 & 93 \\
\hline 000515 & $0431+206$ & -12 & 311 & $\mathrm{E}$ & 53 & - & - & 420 & 78 \\
\hline 000516 & $0321+123$ & -46 & 92 & W & 28 & - & - & 383 & 170 \\
\hline 000516 & $0433+053$ & -58 & 325 & $\mathrm{E}$ & 74 & - & - & 504 & 200 \\
\hline 000517 & $0409+122$ & -62 & 286 & $\mathrm{E}$ & 39 & -20 & 20 & 693 & 35 \\
\hline 000517 & $0431+206$ & -13 & 281 & $\mathrm{E}$ & 46 & 20 & 80 & 428 & 103 \\
\hline 000517 & $0433+053$ & -60 & 310 & $\mathrm{E}$ & 72 & -20 & 20 & 716 & 72 \\
\hline 000518 & $0318+164$ & -10 & 70 & $W$ & 23 & -25 & 25 & 605 & 50 \\
\hline 000518 & $0431+206$ & -13 & 267 & $\mathrm{E}$ & 43 & 10 & 75 & 264 & 124 \\
\hline 000519 & $0318+164$ & -8 & 56 & W & 26 & -25 & 15 & 717 & 141 \\
\hline 000520 & $0318+164$ & -6 & 43 & W & 30 & -20 & 10 & 605 & 73 \\
\hline 000520 & $0431+206$ & -15 & 233 & $\mathrm{E}$ & 35 & -30 & 55 & 592 & 30 \\
\hline \multirow[t]{2}{*}{000521} & $0318+164$ & -5 & 29 & W & 33 & 0 & 80 & 716 & 350 \\
\hline & & -5 & 30 & & & & & 363 & 114 \\
\hline 000522 & $0318+164$ & -4 & 17 & W & 37 & - & - & 315 & 107 \\
\hline 000523 & $0318+164$ & -3 & 4 & W & 40 & -80 & -30 & 316 & 67 \\
\hline \multirow[t]{2}{*}{000524} & $0318+164$ & -2 & 349 & W & 44 & -80 & 0 & 529 & 116 \\
\hline & & -2 & 350 & & & & & 336 & 22 \\
\hline 000525 & $0318+164$ & -1 & 335 & W & 47 & -75 & 10 & 528 & $3 *$ \\
\hline 000526 & $0318+164$ & -1 & 324 & W & 51 & - & - & 360 & 54 \\
\hline
\end{tabular}

al., 2000c, 2002a, b).

Comparisons between in situ and radio-scintillation observations of the solar wind have been carried out in the past, using both measurements of IPS (Behannon et al., 1991; Leinert and Jackson, 1998) and phase scintillation (Spangler and Sakurai, 1995), but the ability of these measurements to resolve streams of solar wind with different velocities was very limited. Quantitative comparisons between high-resolution IPS measurements from long-baseline systems and in situ measurements have been carried out using data from near to solar minimum (Grall et al., 1996; Kojima et al., 1998; Breen et al., 1999), while qualitative comparisons between measurements from a wide range of heliocentric distances at solar maximum have also been presented (e.g. Breen et al., 2000d). In this paper we take advantage of the high-quality long-baseline observations made by EISCAT and compare them with in-ecliptic measurements and out-of-ecliptic measurements from Wind and Ulysses, using a combination of ballistic and MHD mapping. Therefore, this paper represents a first attempt at a quantitative comparison of the large-scale structure of the solar maximum solar wind as revealed by in situ measurements and high-resolution, long-baseline IPS observations.

\section{Datasets}

In this paper we consider data from EISCAT IPS, Wind and Ulysses taken during four periods covering the rise to solar maximum, the period of maximum activity itself and - perhaps - the start of the declining phase:

- 18 August - 14 September 1999 (Carrington rotation 1953)

- 11-28 May 2000 (Carrington rotation 1963)

- 7-13 September 2000 (Carrington rotation 1967)

- 8-31 May 2001 (Carrington rotation 1976)

During August-September 1999 (the Third Whole Sun Month interval, as discussed in Breen at al., 2000d) Ulysses lay at heliographic latitudes of $32^{\circ}-34^{\circ}$ south at a heliocentric distance of almost $1000 R_{S}$. Wind was close to the Earth - at $7^{\circ}$ heliographic latitude and at a heliocentric distance of approximately $215 R_{S}$, and the EISCAT measurements covered distances of 30-80 $R_{S}$ and all heliographic latitudes greater than approximately $-45^{\circ}$ (this limit being imposed by the height of the Sun in the sky towards the end of the arctic summer). During May 2000, Ulysses was further south, 
Table 3. As Table 1 but summarizing EISCAT observations taken between 7 and 13 September 2000 at latitudes within $\pm 15^{\circ}$ of the latitude of Wind, which lay at a mean latitude of $7.24^{\circ}$ during this period, while Ulysses climbed from $-70^{\circ}$ to $-72^{\circ}$, but no EISCAT measurements of these high southern latitudes were successful due to corruption by ground noise. $V_{P}$ is the speed of the solar wind stream across the ray path at the point of closest approach to the Sun (the P-point), and $d V_{\|}$is the variation in speed over the width of the stream (fast or slow) where this parameter was fitted, or the uncertainty in the fitted value of the stream speed (marked * in the latter case). Observations shown in italics may be affected by strong scattering

\begin{tabular}{cccccccccc}
\hline Date & Source & $\begin{array}{c}\text { Latitude } \\
\text { of P-point } \\
\left.\text { (projected to 30 } R_{S}\right)\end{array}$ & $\begin{array}{c}\text { Longitude } \\
\text { of P-point } \\
\left.\text { (projected to 30 } R_{S}\right)\end{array}$ & Limb & Distance of & $\begin{array}{c}\theta_{\text {in }} \\
\text { P-point }\end{array}$ & $\theta_{\text {out }}$ & $V_{P}$ & $d V_{\|}$ \\
\hline 000908 & $1150-003$ & -5 & 216 & $\mathrm{E}$ & 44 & - & - & 246 & 117 \\
000908 & $1229+020$ & 15 & 233 & $\mathrm{E}$ & 75 & - & - & 338 & 69 \\
000909 & $1150-003$ & -5 & 199 & $\mathrm{E}$ & 41 & - & - & 344 & 141 \\
000910 & $1150-003$ & -6 & 184 & $\mathrm{E}$ & 37 & 10 & 40 & 316 & 171 \\
000910 & $1256-057$ & +3 & 217 & $\mathrm{E}$ & 98 & - & - & 372 & 157 \\
000911 & $1150-003$ & -7 & 168 & $\mathrm{E}$ & 34 & -80 & -20 & 423 & 55 \\
000911 & $1256-057$ & +4 & 202 & $\mathrm{E}$ & 94 & - & - & 371 & 141 \\
000912 & $1150-003$ & -8 & 154 & $\mathrm{E}$ & 30 & -80 & -15 & 414 & $5^{*}$ \\
\hline
\end{tabular}

Table 4. As Table 1 but summarizing EISCAT observations taken between 8 and 31 May 2001 at latitudes within $\pm 15^{\circ}$ of the latitude of Wind or Ulysses. Wind lay at a mean latitude of $5^{\circ}$ during this period, while Ulysses passed through the ecliptic during its fast latitude scan. $V_{P}$ is the speed of the solar wind stream across the ray path at the point of closest approach to the Sun (the P-point), and $d V_{\|}$is the variation in speed over the width of the stream (fast or slow) where this parameter was fitted, or the uncertainty in the fitted value of the stream speed (marked * in the latter case). Observations shown in italics may be affected by strong scattering. The observations of $0431+206$ on 24 May and $0521+166$ on 25 May 2001 are probably dominated by the passage of CMEs across the ray path

\begin{tabular}{|c|c|c|c|c|c|c|c|c|c|}
\hline Date & Source & $\begin{array}{c}\text { Latitude } \\
\text { of P-point } \\
\text { (projected to } 30 R_{S} \text { ) }\end{array}$ & $\begin{array}{c}\text { Longitude } \\
\text { of P-point } \\
\text { (projected to } 30 R_{S} \text { ) }\end{array}$ & Limb & $\begin{array}{c}\text { Distance of } \\
\text { P-point }\end{array}$ & $\theta_{\text {in }}$ & $\theta_{\text {out }}$ & $V_{P}$ & $d V_{\|}$ \\
\hline 010521 & $0318+164$ & -5 & 246 & $\mathrm{~W}$ & 34 & 40 & 75 & 315 & 131 \\
\hline 010521 & $0431+206$ & -15 & 84 & $\mathrm{E}$ & 33 & -20 & 60 & 360 & 92 \\
\hline 010522 & $0318+164$ & -3 & 233 & $\mathrm{~W}$ & 38 & 50 & 80 & 324 & 137 \\
\hline 010523 & $0318+164$ & -3 & 219 & W & 41 & 55 & 75 & 381 & 125 \\
\hline 010523 & $0431+206$ & -17 & $\begin{array}{l}54 \\
53\end{array}$ & $\mathrm{E}$ & 30 & 0 & 60 & $\begin{array}{l}728 \\
253\end{array}$ & $\begin{array}{c}2 * \\
101\end{array}$ \\
\hline 010524 & $0318+164$ & -2 & 207 & W & 44 & 60 & 75 & 313 & 171 \\
\hline 010524 & $0431+206$ & -19 & 39 & $E$ & 22 & -20 & 70 & 549 & 16 \\
\hline 010525 & $0318+164$ & -1 & 193 & W & 48 & - & - & 368 & 175 \\
\hline 010525 & $0521+166$ & -28 & 55 & E & 62 & - & - & 324 & 163 \\
\hline 010526 & $0318+164$ & -1 & 179 & W & 51 & -20 & 20 & 475 & $8^{*}$ \\
\hline 010527 & $0318+164$ & 0 & 166 & W & 55 & -15 & 40 & 437 & 98 \\
\hline 010528 & $0318+164$ & 0 & 151 & $\mathrm{~W}$ & 58 & -10 & 40 & 525 & 170 \\
\hline 010529 & $0318+164$ & 1 & 143 & W & 62 & 15 & 80 & 301 & 136 \\
\hline 010530 & $0318+164$ & 1 & 134 & W & 65 & 30 & 80 & 240 & 155 \\
\hline
\end{tabular}

at $-55^{\circ}$ to $-57^{\circ}$ heliographic latitude at a distance close to $750 R_{S}$. Wind was again close to the Earth at a heliographic latitude between $-3^{\circ}$ and $1^{\circ}$. By September 2000, Ulysses had climbed to more than $70^{\circ}$ south (heliographic), and comparisons with EISCAT were extremely difficult, with only a few suitable radio sources above the horizon at such high southern latitudes. During the event, none of the good sources on Ulysses' streams produced usable results, and the comparisons for this period are, therefore, with Wind data only. Ulysses reached its highest southern latitude on 24 November 2000 and by the beginning of May 2001 it was well into its fast latitude scan, passing over the heliographic equator off the west limb at a distance of about $300 R_{S}$ later in the month. Ulysses and Wind, therefore, sampled the solar wind at longitudes $90^{\circ}$ apart during this period, and together with EISCAT observations off the limbs of the Sun near the heliographic equator provide a very important data set for studies of the solar maximum wind near the ecliptic plane. 

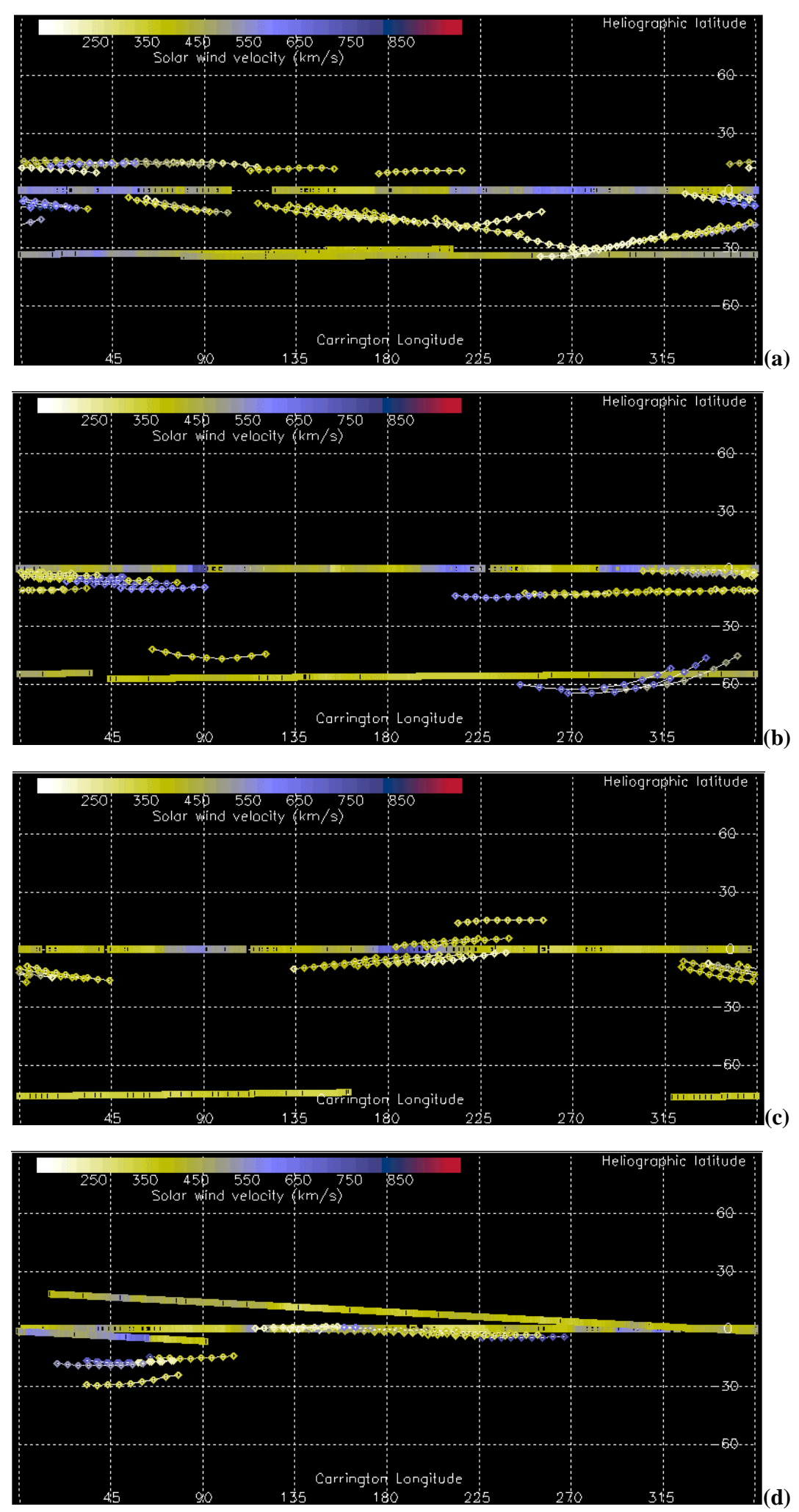

Fig. 2. EISCAT IPS (shown as $\downarrow$ ) and in situ speeds from Wind ( $\square$ ) and Ulysses ( $\square$ ) mapped back to a constant heliocentric distance of $30 R_{S}$. A constant-speed ballistic mapping (using the speeds listed in Tables 1-4) was used for the IPS data, while the in situ data was mapped ballistically using the speeds measured at the spacecraft (except for the Ulysses data shown in (d), which was mapped to $30 R_{S}$ using a MHD model). (a) shows results from CR 1953 (August-September 1999, while results from CR 1963 (May 2000) are shown in (b), CR 1967 (September 2000) in (c) and CR 1976 (May 2001) in (d). 

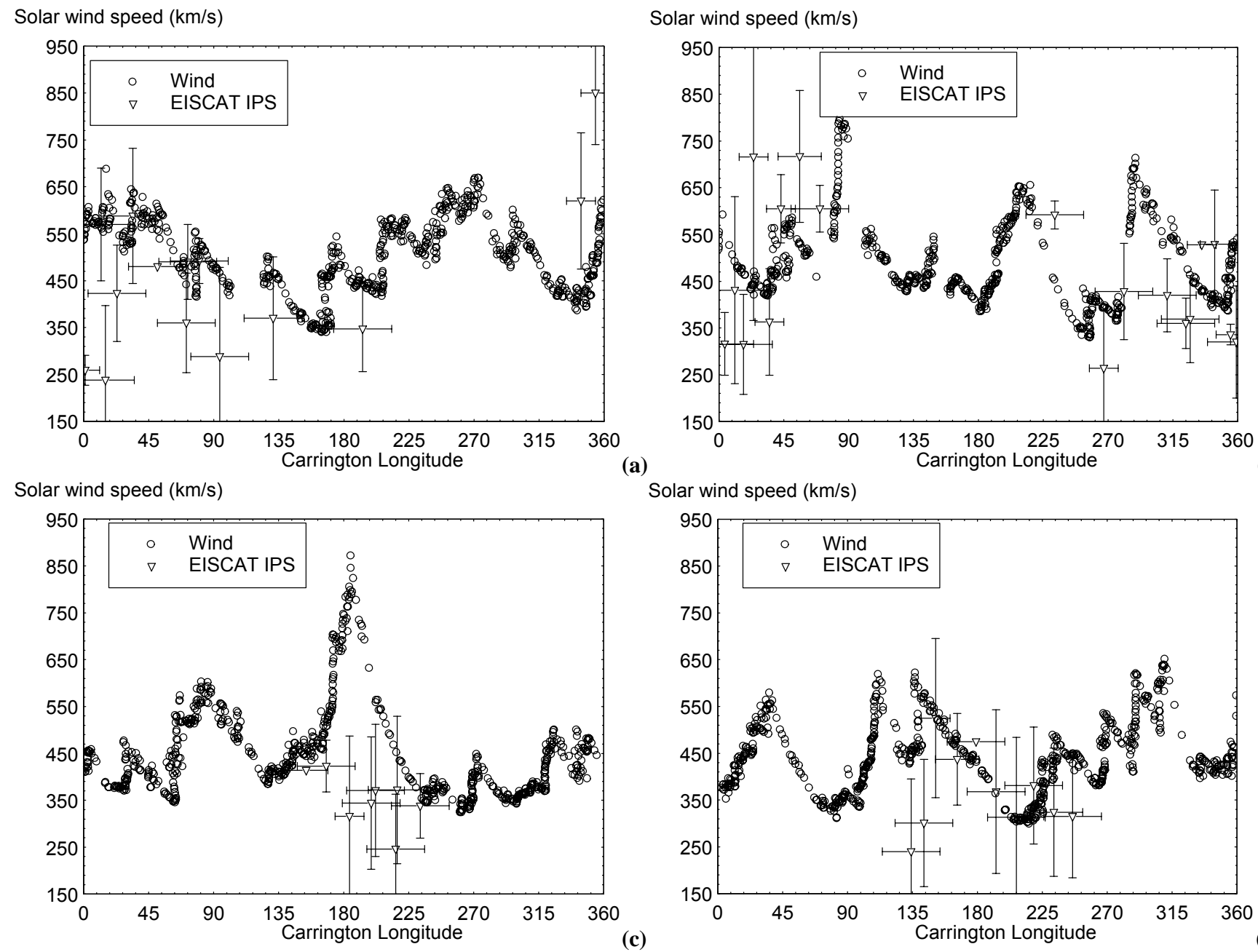

Solar wind speed $(\mathrm{km} / \mathrm{s})$

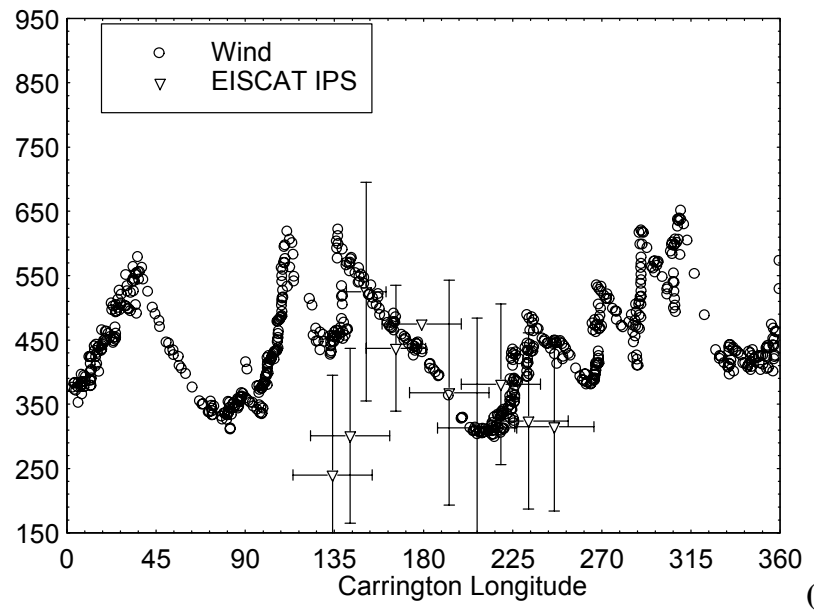

(d)

Fig. 3. Solar wind speeds measured in situ by Wind near the Earth and EISCAT IPS speeds from within $\pm 15^{\circ}$ of the heliographic latitude of Wind mapped ballistically to a uniform heliocentric distance of $30 R_{S}$ and plotted as speed vs. Carrington longitude. (a) shows results from CR 1953 (August-September 1999, while results from CR 1963 (May 2000) are shown in (b), CR 1967 (September 2000) in (c) and CR 1976 (May 2001) in (d). The large vertical bars represent the variation in velocity across the IPS ray path and are not estimates of errors in the measurement.

\subsection{EISCAT IPS observations}

The EISCAT observations made during each of these periods are summarized in Tables $1-4$. The locations of fast and slow streams of solar wind in the ray path for each observation were estimated by projecting the IPS ray paths down to a constant heliocentric distance of $2.5 R_{S}$ using a constantspeed ballistic mapping and overlaying them on white-light maps constructed from LASCO C 2 data. The use of ballistic mapping is not ideal, since there is a strong acceleration of the flow close to the Sun (e.g. Sheeley et al., 1997; Breen et al., 2000b, 2002b) and strong indications of super-radial expansion of fast flow and, moreover, it does not take into account co-rotation effects very close to the Sun. Ballistic mapping does, nonetheless, provide an indication of the location of fast streams, and the method appears to work well (e.g. Breen et al., 1996, 1998, 2000a, d). At solar maximum the uncertainties are larger, but more sophisticated mapping methods, starting from photospheric magnetic field measurements are very difficult to apply during this period due to rapid changes on time scales comparable to a solar rotation - as discussed with reference to the Ulysses and Wind data sets. The mapping method used for the IPS data is illustrated schematically in Fig. 1a. At this stage a "typical" slow wind speed of $400 \mathrm{~km} / \mathrm{s}$ was assumed. Once the position of any likely fast flow had been determined (described by the angles $\theta_{\text {in }}$ and $\left.\theta_{\text {out }}\right)$, the auto- and cross-spectra for the observation were fitted using a 2-D weak scattering model (Grall, 1995; Coles, 1996; Klinglesmith, 1997; Massey, 1998). The ray path was then mapped back to $2.5 R_{S}$ again, this time using the speed suggested by the model fit for the stream occupying the portion of the ray path closest to the Sun, and the fit was repeated with the new values of $\theta_{\text {in }}$ and $\theta_{\text {out }}$. The fit was repeated several times with slightly different starting values to ensure that it was stable and that it did represent a good fit to the observed spectra. Once a satisfactory fit was obtained, 


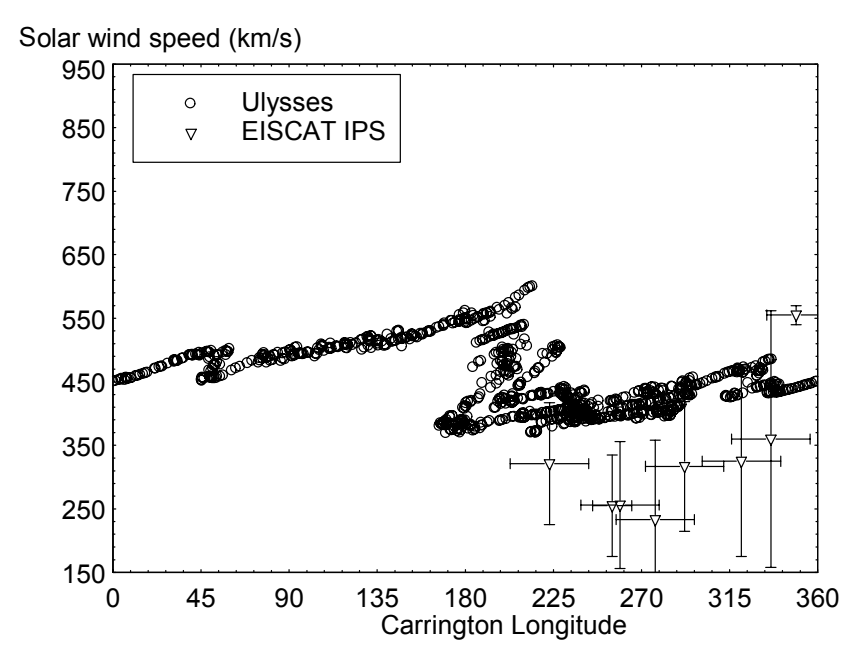

Solar wind speed $(\mathrm{km} / \mathrm{s})$

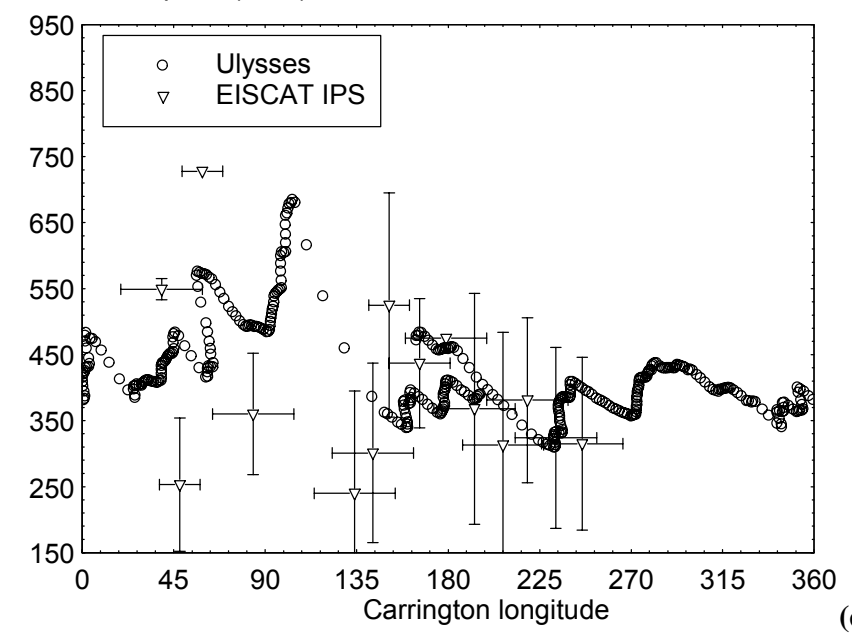

Solar wind speed $(\mathrm{km} / \mathrm{s})$

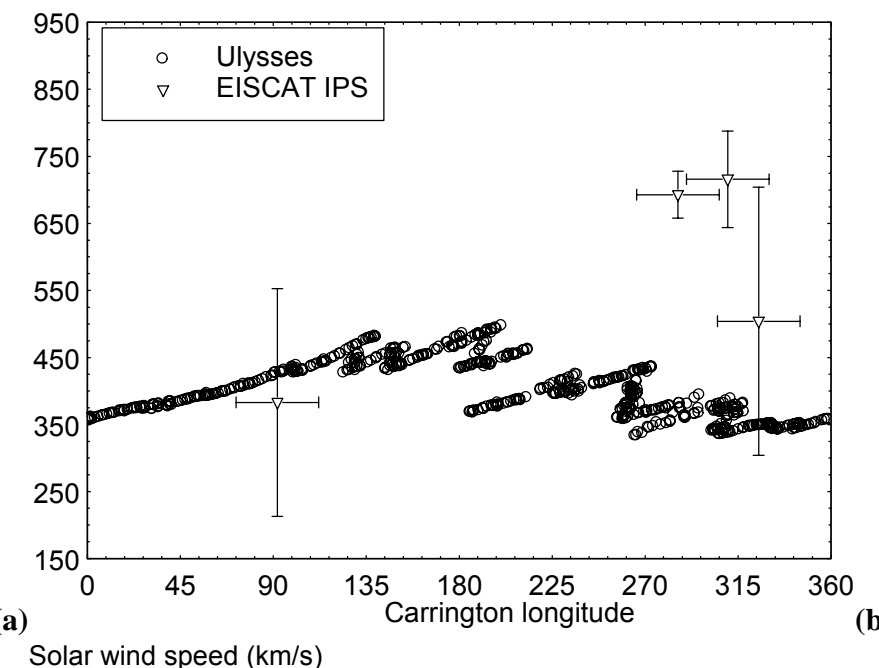

(b)

Solar wind speed $(\mathrm{km} / \mathrm{s})$

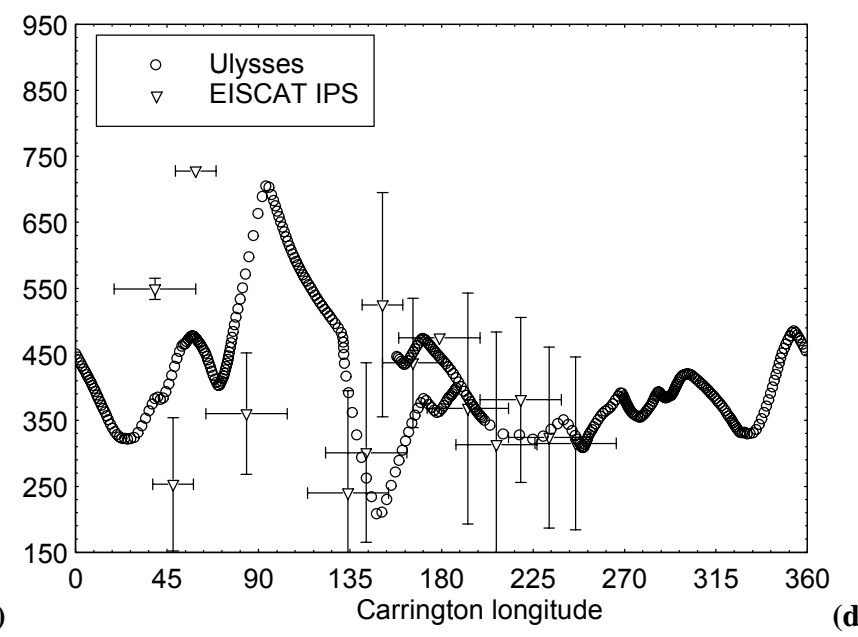

(d)

Fig. 4. Ulysses in situ speed measurements and EISCAT IPS speeds from within $\pm 15^{\circ}$ of any latitude of Ulysses over each interval, mapped to a uniform heliocentric distance of $30 R_{S}$ using either a ballistic projection or (d) a hybrid MHD/ballistic mapping method and plotted as speed vs. Carrington longitude. (a) shows results from CR 1953 (August-September 1999), while results from CR 1963 (May 2000 ) are shown in (b) and CR 1976 (May 2001) in (c) (ballistically-mapped Ulysses data) and (d) (MHD/ballistic mapping). There were no good EISCAT observations at sufficiently high southern latitudes for comparison with Ulysses during CR 1967. The large vertical bars represent the variation in velocity across the IPS ray path and are not estimates of errors in the measurement.

the IPS ray paths were mapped to a constant heliocentric distance of $30 R_{S}$ using a constant-speed ballistic model, with the speed used being that suggested by the analysis model fit for the portion of the ray path closest to the Sun. The results are summarised in Tables 1-4: in all cases the speeds used for mapping (VP) are listed in column 9 and the uncertainties in the speed across this region of the ray path (between $\theta_{\text {in }}$ and $\left.\theta_{\text {out }}\right)$ as $d V_{\|}$in column 10 . In most observations the uncertainty in speed is likely to be dominated by the real variation in solar wind speed over the extended ray path.

\subsection{In situ data from Wind and Ulysses}

The in situ plasma data used in this study were taken by the Solar Wind Experiment (SWE) on the Wind spacecraft (Ogilvie et al., 1995) and by the Solar Wind Observations
Over the Poles of the Sun (SWOOPS) plasma experiment on Ulysses (Bame et al., 1992). These data were also mapped back to a constant heliocentric distance of $30 R_{S}$. Since the distances involved are much greater, it was considered best to perform the mapping using an MHD model based on the photospheric magnetic field developed at SAIC (e.g. Mikic and Linker, 1996; Linker et al., 1999) and recently extended to greater distances from the Sun (e.g. Riley et al., 1999, 2001). This approach divides the modelling into two regions: the solar corona (1-30 $R_{S}$ solar radii) and the heliosphere $\left(30-1075 R_{S}\right)$. The solar coronal component of the model is driven by measurements of the line-of-sight magnetic field in the photosphere, such as those produced by the National Solar Observatory at Kitt Peak, while inputs to the heliospheric component of the model are derived from the output of the solar coronal model. Solar wind plasma and magnetic field 

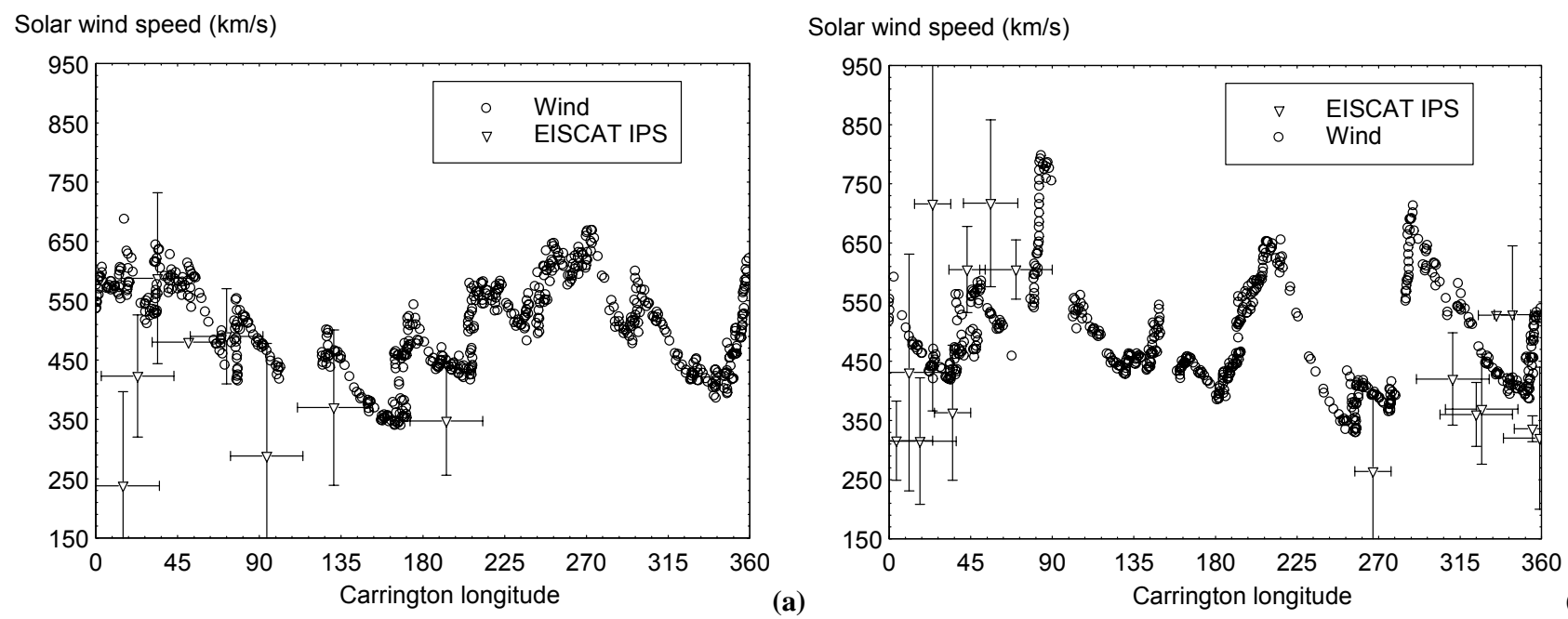

Solar wind speed $(\mathrm{km} / \mathrm{s})$

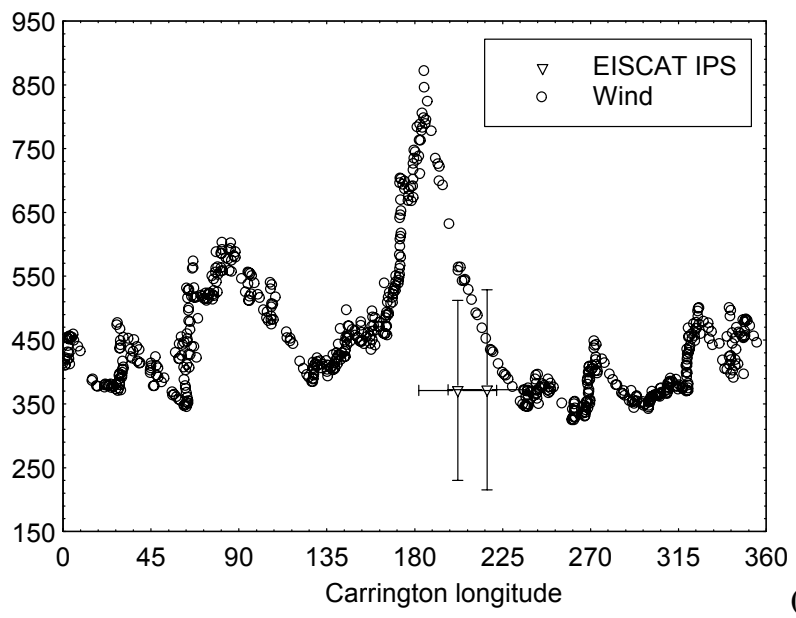

Solar wind speed $(\mathrm{km} / \mathrm{s})$

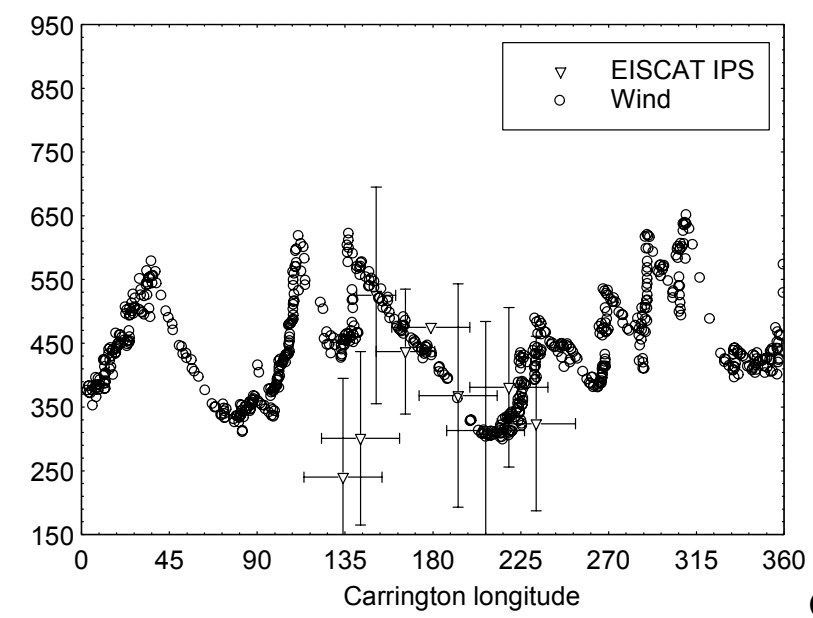

(d)

Fig. 5. Solar wind speeds measured in situ by Wind near the Earth and EISCAT IPS speeds from within $\pm 10^{\circ}$ of the heliographic latitude of Wind mapped ballistically to a uniform heliocentric distance of $30 R_{S}$ and plotted as speed vs. Carrington longitude. (a) shows results from CR 1953 (August-September 1999, while results from CR 1963 (May 2000) are shown in (b), CR 1967 (September 2000) in (c) and CR 1976 (May 2001) in (d). The large vertical bars represent the variation in velocity across the IPS ray path and are not estimates of error in the measurement.

parameters at $1 \mathrm{AU}$ and beyond can, therefore, be derived from the observed photospheric magnetic field. In this study the SAIC MHD model was used to trace streams of solar wind inwards from the spacecraft measurements to $0.75 R_{S}$ (0.35 AU), with ballistic mapping used to map the streams in to $30 R_{S}$. Since interaction between the streams is more important at larger distances, the use of ballistic mapping in the inner regions of the solar wind (though still outside the acceleration region) is acceptable.

Unfortunately, during late 1999 and 2000, the solar magnetic field was evolving so rapidly that these equilibrium models were not appropriate for this study and so we were compelled to use simple ballistic mapping for in situ data from Carrington rotations CR 1953 (August-September 1999), CR 1963 (May 2000) and CR 1967 (September 2000). The speeds used were taken from the in situ data and were assumed to be constant. It did, however, prove possible to use the MHD model for data from CR 1976 (May 2001).

\section{Results}

IPS and in situ speeds mapped to $30 R_{S}$ are plotted on heliographic latitude/Carrington longitude maps for the four intervals studied in Fig. 2. In each case the central $40^{\circ}$ of each IPS ray path is plotted (from $\theta_{\text {in }}=-20^{\circ}$ to $\theta_{\text {out }}=20^{\circ}$, a region that contributes approximately $50 \%$ of the observed scintillation in a single-stream solar wind), together with the paths traced out by Wind and Ulysses. In all cases the speeds are shown colour-coded. Figure 3 displays the variation with longitude of solar wind speeds measured by EISCAT IPS at latitudes within $15^{\circ}$ of Wind and by the Wind spacecraft itself for the same four intervals, while Fig. 4 repeats the comparison for IPS and Ulysses measurements. In Figs. 5 and 6 the comparison is restricted to IPS measurements from latitudes within $10^{\circ}$ of Wind (Fig. 5) and Ulysses (Fig. 6). In all of Figs. 2-6 results from CR 1953 are presented in (a), with CR 1963 results shown in (b), CR 1967 in (c) and CR 1976 

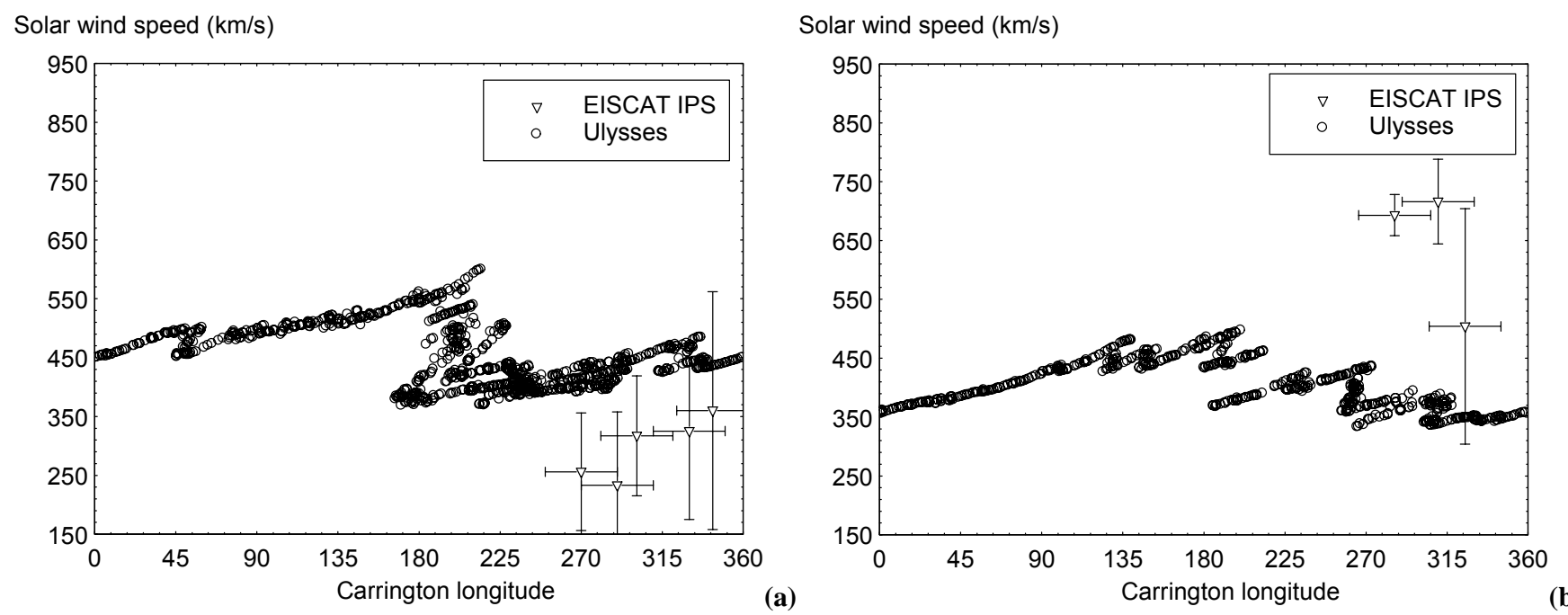

Solar wind speed $(\mathrm{km} / \mathrm{s})$

Solar wind speed $(\mathrm{km} / \mathrm{s})$
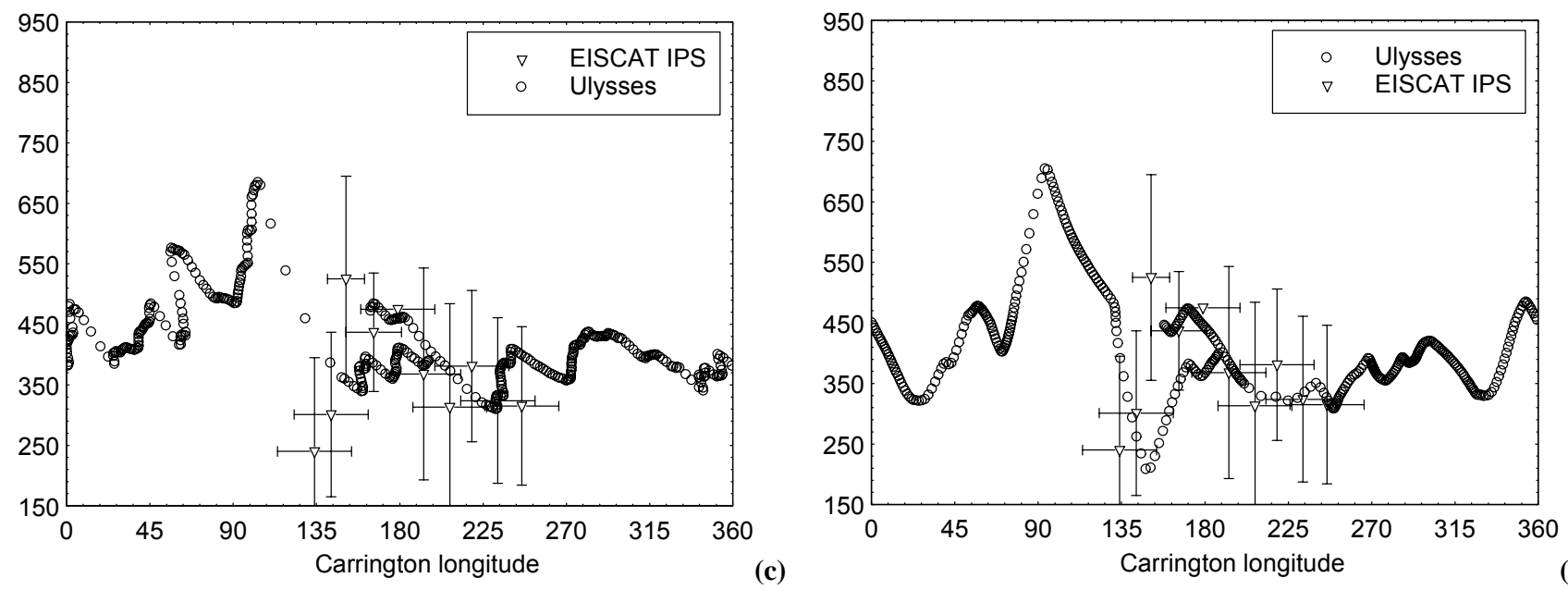

(d)

Fig. 6. Ulysses in situ speed measurements and EISCAT IPS speeds from within $\pm 10^{\circ}$ of the actual latitude of Ulysses over each interval, mapped to a uniform heliocentric distance of $30 R_{S}$ using either a ballistic projection or (d) a MHD mapping and plotted as speed vs. Carrington longitude. (a) shows results from CR 1953 (August-September 1999, while results from CR 1963 (May 2000) are shown in (b) and CR 1976 (May 2001) in (c) (ballistic mapping) and (d) (MHD/ballistic mapping). There were no good EISCAT observations at sufficiently high southern latitudes for comparison with Ulysses during CR 1967. The large vertical bars represent the variation in velocity across the IPS ray path and are not estimates of errors in the measurement.

in $(\mathrm{d})$.

\subsection{CR 1953, 18 August - 14 September 1999}

There was good agreement between the solar wind speeds measured by EISCAT at latitudes close to that of Wind $\left(7^{\circ} \mathrm{N}\right)$ and those recorded in situ by Wind during this period (Fig. 2a), though EISCAT did suggest significantly higher speeds near $0^{\circ}$ longitude, together with significantly lower speeds close to $20^{\circ}$ and $340^{\circ}$ longitude, as shown in Fig. 3a. Apart from one observation centred on $24^{\circ}$ longitude $(1042+120$ on 20 September 1999, with a P-point at $11^{\circ} \mathrm{N}$ latitude), the IPS speeds follow the general trend of the in situ data well, but appear on closer inspection to suggest a larger variation in flow speed. The large vertical "error" bars in the IPS speeds do not, in most cases, represent the statistical error in the IPS fit in the estimated bulk flow speed but instead provide an estimate of the variation in solar wind speed over the extended IPS ray path. While the bulk flow speed estimated by the analysis model represents the average speed across that part of the ray path containing the P-point, weighted by $R^{-4}$ and thus heavily biased towards the flow speed across the P-point, the spread in speeds will be considerably enhanced if there are significant gradients in speed present - for example, if the ray path passes through regions of slow wind with different intrinsic speeds. It is important to note that the spread in speeds represented by $d V_{\|}$may not be symmetrical about the estimated bulk flow speed, but since there is no easy way of determining this asymmetry, we have plotted the points in $V \pm d V_{\|}$form.

During this period Wind lay at latitudes between $6.76^{\circ}$ and $7.22^{\circ}$ heliographic latitude, and when the comparison was 
Table 5. Comparison of speeds determined from EISCAT IPS data with in situ speeds measured by Wind and Ulysses. The IPS observations all had P-points which lay within $\pm 10^{\circ}$ of the heliographic equator (comparisons with Wind) or the latitude of Ulysses, while the in situ speeds used for the comparison come from the range of longitudes that would contribute $50 \%$ of the observed scintillation in the IPS measurement (or the width of the stream across the P-point, whichever is less). All speeds are in $\mathrm{km} / \mathrm{s}$, and the spread in speeds is given as the difference between the minimum and maximum speeds seen by IPS and the spread within in situ velocities over the longitude range for which there are IPS results. $\left\langle V_{\text {IPS }}\right\rangle,\left\langle V_{\text {Wind }}\right\rangle$ and $\left\langle V_{\text {Ulysses }}\right\rangle$ are all calculated as unweighted means, as the variation in velocity is not normally due to statistical error in measurement or fitting. Data marked * may be affected by the passage of a coronal mass ejection. Data shown in italic type correspond to observations of slow streams which may lie within the acceleration region of the slow solar wind or may be affected by the onset of strong scattering. In these cases values of $\left\langle V_{\text {IPS }}\right\rangle$, Spread in $V_{\text {IPS }}$ and $\sigma\left(V_{\text {IPS }}\right)$ are given for cases when these points are included and when neglected. The IPS measurements made during CR 1963 came from slightly higher latitudes than those from Ulysses and show high-speed wind that Ulysses did not encounter. The Wind observations of the longitudes for which IPS observations near the heliographic equator are available during CR 1967 were dominated by the passage of a coronal mass ejection, apparent in the enhanced mean speed and large variation in solar wind speed measured in situ

\begin{tabular}{|c|c|c|c|c|c|c|c|c|c|c|}
\hline $\begin{array}{l}\text { Carrington } \\
\text { rotation }\end{array}$ & $V_{\text {IPS }}$ & $d V_{\| \mathrm{IPS}}$ & $V_{\text {Wind }}$ & $\sigma\left(V_{\text {Wind }}\right)$ & $\left\langle V_{\text {IPS }}\right\rangle$ & $\left\langle V_{\text {Wind }}\right\rangle$ & Spread in & $\begin{array}{c}\sigma\left(V_{\text {IPS }}\right) \\
V_{\text {IPS }}\end{array}$ & Spread in & $\begin{array}{c}\sigma\left(V_{\text {Wind }}\right) \\
V_{\text {Wind }}\end{array}$ \\
\hline \multirow[t]{8}{*}{ CR 1953} & 288 & 190 & 477 & 38 & $403 *$ & 522 & $350 *$ & $115^{*}$ & 144 & 57 \\
\hline & 347 & 91 & 477 & 48 & 427 & & 300 & 101 & & \\
\hline & 370 & 131 & 434 & 41 & & & & & & \\
\hline & 480 & 5 & 569 & 43 & & & & & & \\
\hline & 588 & 144 & 578 & 33 & & & & & & \\
\hline & 423 & 103 & 578 & 34 & & & & & & \\
\hline & 490 & 80 & 498 & 45 & & & & & & \\
\hline & $238 *$ & 151 & 568 & 31 & & & & & & \\
\hline \multirow[t]{15}{*}{ CR 1963} & 431 & 200 & 462 & 43 & 462 & 483 & 402 & 144 & 205 & 53 \\
\hline & 320 & 120 & 453 & 48 & 452 & & 402 & 144 & & \\
\hline & 369 & 93 & 465 & 57 & & & & & & \\
\hline & 420 & 78 & 557 & 62 & & & & & & \\
\hline & 605 & 50 & 627 & 102 & & & & & & \\
\hline & 717 & 141 & 523 & 38 & & & & & & \\
\hline & 605 & 73 & 503 & 50 & & & & & & \\
\hline & 716 & 350 & 491 & 53 & & & & & & \\
\hline & 363 & 114 & 456 & 19 & & & & & & \\
\hline & 315 & 107 & 461 & 39 & & & & & & \\
\hline & 316 & 67 & 458 & 47 & & & & & & \\
\hline & 529 & 116 & 465 & 52 & & & & & & \\
\hline & 336 & 22 & 422 & 18 & & & & & & \\
\hline & 528 & 3 & 428 & 27 & & & & & & \\
\hline & 360 & 54 & 471 & 57 & & & & & & \\
\hline \multirow[t]{2}{*}{ CR 1967} & 371 & 157 & 455 & 87 & 372 & 522 & 1 & - & 195 & - \\
\hline & 372 & 141 & 650 & 143 & & & & & & \\
\hline \multirow[t]{9}{*}{ CR 1976} & 324 & 137 & 390 & 55 & 374 & 454 & 285 & 92 & 204 & 76 \\
\hline & 381 & 125 & 366 & 56 & & & & & & \\
\hline & 313 & 171 & 333 & 28 & & & & & & \\
\hline & 368 & 175 & 537 & 61 & & & & & & \\
\hline & 475 & 8 & 436 & 40 & & & & & & \\
\hline & 437 & 98 & 471 & 31 & & & & & & \\
\hline & 525 & 170 & 536 & 27 & & & & & & \\
\hline & 301 & 136 & 508 & 55 & & & & & & \\
\hline & 240 & 155 & 510 & 58 & & & & & & \\
\hline
\end{tabular}

restricted to observations with P-points, which lay between $17^{\circ}$ and $-3^{\circ}$ heliographic latitude, the agreement between IPS and in situ speeds improved significantly, as shown in Fig. 5a. Speeds at Wind were generally higher than suggested by the IPS results, and there appeared to be rather more variation in speeds seen by IPS than in those measured by Wind. There were fewer IPS observations at the latitude of Ulysses, but all measurements from within $15^{\circ}$ latitude of Ulysses are compared with the corresponding in situ speeds in Fig. $4 \mathrm{a}$, while the four measurements from within $10^{\circ}$ of 
Table 5. continued ...

\begin{tabular}{|c|c|c|c|c|c|c|c|c|c|c|}
\hline $\begin{array}{l}\text { Carrington } \\
\text { rotation }\end{array}$ & $V_{\text {IPS }}$ & $d V_{\| \mathrm{IPS}}$ & $V_{\text {Ulysses }}$ & $\sigma\left(V_{\text {Ulysses }}\right)$ & $\left\langle V_{\text {IPS }}\right\rangle$ & $\left\langle V_{\text {Ulysses }}\right\rangle$ & Spread in & $\begin{array}{c}\sigma\left(V_{\text {IPS }}\right) \\
V_{\text {IPS }}\end{array}$ & Spread in & $\begin{array}{c}\sigma\left(V_{\text {Ulysses }}\right) \\
V_{\text {Ulysses }}\end{array}$ \\
\hline \multirow[t]{5}{*}{ CR1953 } & 360 & 202 & 405 & 8 & 298 & 402 & 127 & 52 & 7 & 3 \\
\hline & 325 & 150 & 402 & 7 & 343 & & 35 & - & & \\
\hline & 317 & 102 & 405 & 5 & & & & & & \\
\hline & 233 & 125 & 402 & 6 & & & & & & \\
\hline & 256 & 100 & 398 & 7 & & & & & & \\
\hline \multirow[t]{3}{*}{ CR1963 } & 504 & 200 & 352 & 12 & 638 & 358 & 212 & 116 & 14 & 7 \\
\hline & 716 & 72 & 357 & 16 & & & & & & \\
\hline & 693 & 35 & 366 & 23 & & & & & & \\
\hline \multirow[t]{10}{*}{ CR 1976} & 315 & 131 & 350 & 27 & 368 & 364 & 285 & 140 & 88 & 29 \\
\hline & 324 & 137 & 328 & 15 & 374 & & & & 91 & \\
\hline & 381 & 125 & 33310 & & & & & & & \\
\hline & 313 & 171 & 346 & 16 & & & & & & \\
\hline & 368 & 175 & 383 & 30 & & & & & & \\
\hline & 475 & 8 & 404 & 42 & & & & & & \\
\hline & 437 & 98 & 409 & 51 & & & & & & \\
\hline & 525 & 170 & 357 & 91 & & & & & & \\
\hline & 301 & 136 & 344 & 88 & & & & & & \\
\hline & 240 & 155 & 386 & 98 & & & & & & \\
\hline
\end{tabular}

the latitude of Ulysses and the corresponding Ulysses speeds are shown in Fig. 6a. In all cases the speeds seen by Ulysses were greater than those determined from IPS data, while the spread in flow speed $d V_{\|}$required to fit the IPS measurements suggested a greater variation in flow speed than was apparent in the in situ measurements that corresponded to the same longitudes at $30 R_{S}$.

Table 5 summarises the speed results for the four intervals. The results from CR 1953 show that IPS speeds at 27-100 $R_{S}$ are, on average, slower than those measured near $215 R_{S}$ by Wind and near $1000 R_{S}$ by Ulysses. There is considerably more variation in the IPS speeds than those measured in situ (the spread and standard deviations of IPS speeds being about 3 times greater than the values for Wind measurements and considerably larger than the variation seen at Ulysses). IPS observations suggested enhanced flow speeds at the same longitudes as Wind measurements showed increases in solar wind speed, but some of the IPS results (near $0^{\circ}$ Carrington longitude) indicated considerably faster flow than was seen in situ. Conversely, IPS suggested slower flow speeds than were measured in situ at longitudes where the Wind data showed the slowest flow. It should be noted that the slowest speeds observed by EISCAT are for those slow wind observations closest to the Sun - it is likely that these observations are within the slow wind acceleration region and can, therefore, be expected to be show slower flow than measured in situ. When these points (denoted by italic type in Table 5) are eliminated from the comparison with in situ velocities, the variation in IPS speeds is still considerably greater than measured in situ, showing that the discrepancy between the data sets cannot be solely due to the acceleration of the slow wind.

\subsection{CR 1963, 11 - 28 May 2000}

These data were taken near the peak of solar maximum, when the configuration of the corona and solar wind changed considerably in the course of a solar rotation. Wind lay at heliographic latitudes between $-3^{\circ}$ and $1^{\circ}$, while Ulysses was climbing towards its southern polar pass and lay between $-55^{\circ}$ and $-57^{\circ}$ latitude. The observations are summarised in Fig. 2b, while the variations with longitude of speeds determined from IPS observations and measured in situ by Wind and Ulysses are compared in Figs. $3 \mathrm{~b}$ and $4 \mathrm{~b}$, respectively.

When all IPS observations close in latitude to Wind were compared with ballistically-mapped Wind data (Fig. 3b), the agreement was fairly good, with lower-than-average flow speeds seen at similar longitudes in both data sets. When the IPS data set was restricted to observations from within $\pm 10^{\circ}$ of the latitude of Wind (Fig. 5b), the agreement between the data sets improved significantly. The IPS observations again suggest a considerably greater range of solar wind speeds than the Wind measurements, particularly between $0^{\circ}$ and $90^{\circ}$ Carrington longitude, where sharp velocity gradients were present in both data sets. The mean flow speed suggested by IPS data was higher over this rotation, but this was due to the high flow speeds near $45^{\circ}$ longitude. The variation in IPS speeds was between 2 and 2.7 times as great as seen in the Wind measurements. These results, together with those from CR 1953, suggest that longitudinal gradients in velocity may be steeper closer to the Sun.

The agreement between the IPS results from within $15^{\circ}$ 

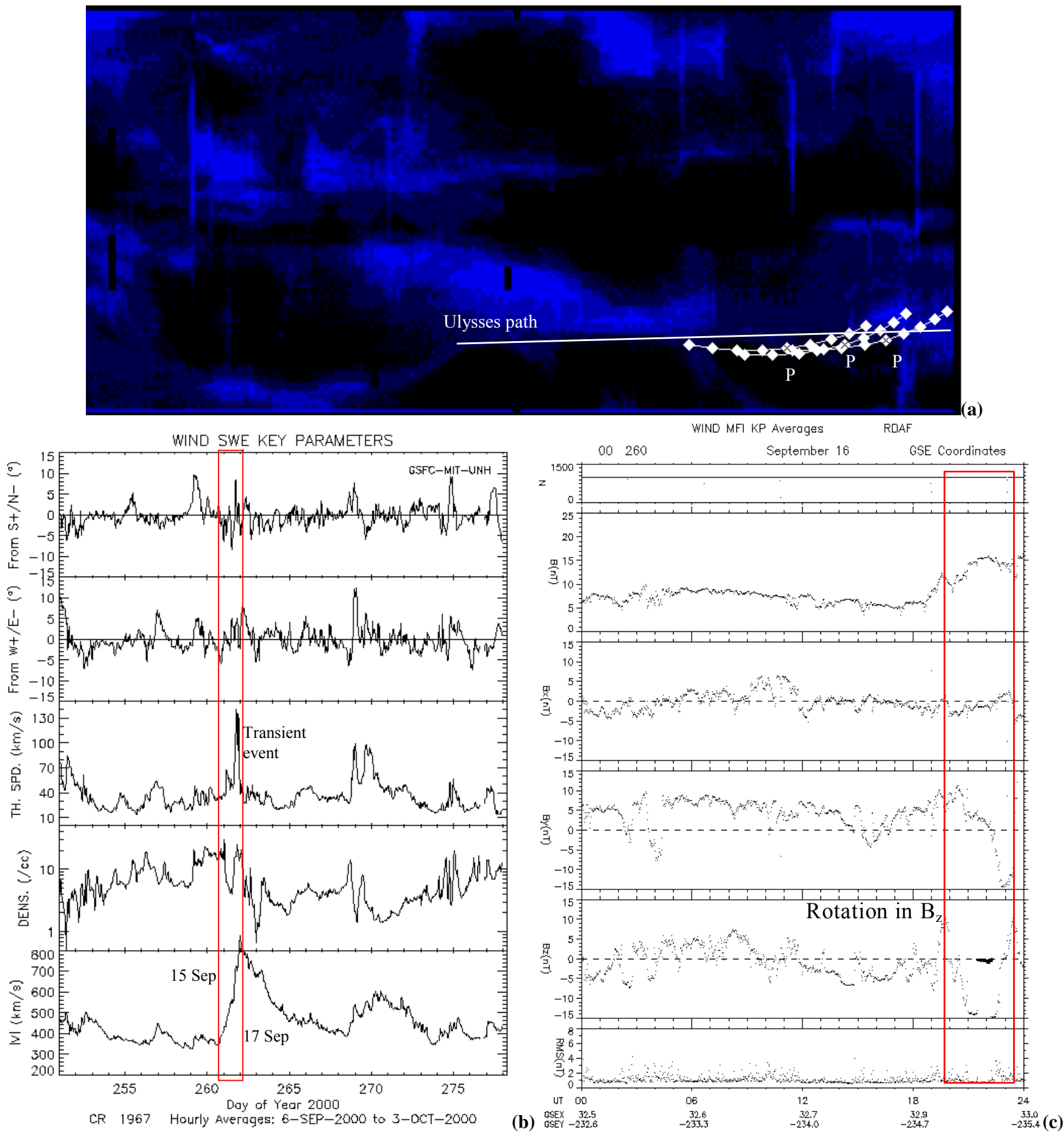

Fig. 7. (a) Ray-paths for the EISCAT observations of 0433+053 on 16 May 2000, 0409+122 on 17 May 2000 and $0433+053$ on 17 May 2000 which lay close in latitude and longitude to the path traced out by Ulysses but indicated considerably higher flow speeds than were observed in situ. Here the ray paths are mapped back ballistically to $2.5 R_{S}$ and overlaid on a map of white-light intensity constructed from LASCO C2 data taken off the east limb of the Sun during CR 1963. The path traced by Ulysses across these latitudes and longitudes during the month is overlaid. The P-points for the three EISCAT observations lie slightly poleward of the Ulysses trajectory and appear to be above an extension of the southern polar coronal hole, whereas Ulysses lay above a region of brighter corona. The EISCAT observations were, therefore, heavily influenced by fast flow from the coronal hole, which Ulysses did not pass through. (b) In situ plasma data from Wind SWE during CR 1967 (September 2000). The high-speed event shown in Figs. 3c and 5c is conspicuous, and in this plot it can be seen that the high-speed peak on 16 September 2000 occurs at the same time as a sharp increase in thermal speed and a rapid change in the north-south component of velocity. (c) Magnetic field data from Wind MFI during 16 September 2000. A strong rotation in magnetic field lasting 3-4 h is seen in the north-south component of the interplanetary magnetic field. This strongly suggests the passage of a coronal mass ejection over Wind during 16 September 2000. 
of the Ulysses trajectory, projected back ballistically to $30 R_{S}$, and the corresponding Ulysses speeds was very poor (Fig. 4b), with EISCAT suggesting much greater flow speeds at longitudes between $270^{\circ}$ and $360^{\circ}$. In contrast to the cases discussed up until now, this agreement did not improve when only IPS observations from within $10^{\circ}$ of the latitude of Ulysses were considered - in fact, the match became significantly worse since only the three fastest speeds observed by EISCAT remained. Careful comparison of the positions of the ray paths for the EISCAT observations and the trajectory of Ulysses (both mapped back ballistically to $2.5 R_{S}$ ) with coronal white-light intensities showed that the P-points for the EISCAT observations lay at slightly higher southern latitudes than the Ulysses path, and that this small increase in latitude was enough to place the EISCAT ray paths above an equatorwards extension of the southern polar coronal hole, as shown in Fig. 7a. The EISCAT observations were, therefore, dominated by flow from the coronal hole and the hole boundary, which would explain the high mean velocities and the large spread in velocity in one of the observations. The Ulysses measurements, by contrast, map back to above the bright corona, where slow flow would be expected - and was indeed observed.

\subsection{CR 1967, 7 - 13 September 2000}

During the period of these observations, the Sun was very close to maximum activity, with considerable changes observed in coronal structure over the two weeks of EISCAT observations. The observations are summarised in Fig. 2c, and it is apparent that during this period Ulysses lay further south than any of the good-quality IPS observations - comparisons of IPS and in situ velocities are, therefore, restricted to EISCAT and Wind for this period.

Figure $3 \mathrm{c}$ compares solar wind speeds measured in situ at Wind which have been mapped back ballistically to $30 R_{S}$, with velocities estimated from IPS observations at similar latitudes. There is good agreement between the measurements at longitudes between $340^{\circ}$ and $40^{\circ}$, but the most dramatic feature seen by Wind - the very sharp enhancement in flow speed near $190^{\circ}$ longitude - is not seen in EISCAT data from the same longitude range. During this interval, Wind lay at latitudes between $7.25^{\circ}$ and $7.23^{\circ}$. When the velocities derived from IPS observations for which the P-point lay within $\pm 10^{\circ}$ of the actual latitude of Wind are compared with the corresponding speeds measured in situ at Wind the disagreement remains obvious. Since the IPS measurements, which covered the longitude range where Wind detected fast flow lay very close to Wind in latitude, it does not seem possible to explain the much higher speeds seen by Wind in terms of their origin in a latitudinally-narrow near-equatorial coronal hole. Furthermore, no such feature is present in white-light coronal maps constructed from data taken over CR 1967. Examination of the plasma and field measurements made by Wind during this period reveals a sharp change in direction of the north-south component of the interplanetary magnetic field (Fig. 7c) at the same time that enhanced flow speeds appeared (Fig. 7b). The field rotation and the peak in wind speed were observed at Wind on 17 September, and there had been a series of Halo events observed by LASCO between 15:26 UT on 15 September and 05:26 UT on 16 September. Wind observations on 16-17 September 2000 showed a maximum speed of $\sim 900 \mathrm{~km} / \mathrm{s}$, and a CME moving out from the Sun at this speed would have taken $\sim 46 \mathrm{~h}$ to reach $1 \mathrm{AU}$. The high flow speeds seen by Wind, which were not present in the IPS record, therefore, almost certainly represent the passage of a coronal mass ejection, which was not observed by EISCAT. Away from the CME event there was good agreement between the EISCAT and in situ measurements.

\subsection{CR 1976, 8 - 31 May 2001}

This interval covered the Ulysses ecliptic pass, with Ulysses sweeping through from a latitude of $-6^{\circ}$ at the start of the interval to $+18^{\circ}$ by the end of May. Wind lay at $-4^{\circ}$ at the start of the month, but its latitude increased through the interval of study. Ulysses was at its closest to the Sun during May 2001, lying about $1.35 \mathrm{AU}\left(\sim 290 R_{S}\right)$ off the west limb of the Sun, while Wind was always close to the Sun-Earth line and, therefore, sampled the solar wind at longitudes $90^{\circ}$ away from Ulysses.

The observations from May 2001 are summarised in Fig. 2d. During this interval the magnetic configuration of the Sun had become significantly simpler than it had been over the peak of solar maximum, and it was possible to compare results obtained when the SAIC MHD/ballistic hybrid model (Riley et al., 2001) was used to map the in situ measurements back to $30 R_{S}$ with those obtained from ballistic mapping. IPS observations, coming from regions much closer to the $30 R_{S}$ reference distance than was the case with the in situ measurements, were still mapped ballistically, as were the Wind data. Figure $3 \mathrm{~d}$ shows the speeds measured by Wind, mapped back to $30 R_{S}$, together with IPS speeds from similar latitudes. The agreement is generally good, although IPS measurements suggest that the narrow, slow stream near $130^{\circ}$ longitude may have been more distinct close to the Sun than at $1 \mathrm{AU}$. Once again, large values of $d V_{\|}$were needed to fit IPS observations of regions where Wind saw steep longitudinal gradients in outflow speed. IPS observations within $10^{\circ}$ of the latitude of Wind are compared with the Wind results in Fig. 5d - the results are little different from those shown in Fig. 3d, so again there is good agreement, with the region of decreasing wind speed between $140^{\circ}$ and $200^{\circ}$ Carrington longitude showing particularly good agreement. The spread of IPS speeds through this period was smaller than for the corresponding measurements from Wind, since the IPS observations did not detect the fast stream seen by Wind near $140^{\circ}$ longitude. The standard deviation of the IPS speeds was, however, greater than for the corresponding Wind measurements. The agreement between IPS wind speeds and those measured by Ulysses was impressive, even when all EISCAT observations from within $\pm 15^{\circ}$ of the helioequator were considered (Figs. 4c, d). When only obser- 
vations for which the P-point lay at a latitude within $\pm 10^{\circ}$ of the Ulysses latitude were considered, then a very good match between the regions of lower and higher speed flow detected by EISCAT and Ulysses was apparent (Figs. 6c, d). In both cases there was substantially better agreement between the MHD-mapped Ulysses data and the IPS observations (as shown in Figs. 3d and 6d) than was seen with ballistically-mapped Ulysses data (Figs. 4c and 6c).

IPS observations indicated enhanced flow speeds near $160^{\circ}$ longitude which were significantly greater than the corresponding in situ measurements, although the lowest flow speeds (near $140^{\circ}$ longitude) were similar in both data sets. Away from the region, between longitudes of $135^{\circ}$ and $180^{\circ}$, where there were substantial longitudinal gradients in velocity, the speeds seen by EISCAT and Ulysses were similar, although the values of $d V_{\|}$required to fit the observed IPS spectra suggest that there might be more variation in flow speed at $30-70 R_{S}$ than is apparent in the in situ data. The velocities are summarised in Table 5, and the larger variation in IPS speeds is clearly apparent.

\section{Discussion: mapping methods and uncertainties in results}

In this paper we have employed two methods of mapping observations of different regions of the solar wind to a single reference distance of $30 R_{S}$ - constant-velocity ballistic mapping and a hybrid MHD/ballistic model developed at SAIC (Riley et al., 2001). The use of any mapping method inevitably introduces some uncertainties, and an additional level of uncertainly is introduced by the method used to determine which regions of the ray path of a given IPS observation are immersed in fast and slow flow. This is done by ballistically mapping the IPS ray path down to $2.5 R_{S}$ at constant velocity and overlaying it on a map of white-light intensity in the corona - dark regions of the corona are assumed to map to fast flow and bright regions to slow. Although the solar wind does not have a constant velocity close to the Sun (the fast wind accelerating out to reach its cruising speed by $10-15 R_{S}$ and the slow wind accelerating strongly out to $25-$ $30 R_{S}$, with slow acceleration at greater distances) the uncertainty in source longitude is relatively small, since most of the acceleration takes place close to the Sun where the angle of the Parker spiral is small. The effects of non-radial expansion of the solar wind should also be small outside $2.5 R_{S}$, so that the largest uncertainty introduced in this stage of analysis is that due to the changes in coronal structure over the 27 days needed to create the white-light map. As small regions of fast flow can open and close on time scales of well under a solar rotation at solar maximum and the location of streamers change at comparable speeds, the location of regions of fast and slow wind estimated from the maps may not be identical to those prevailing at the time when the observation is being analysed. We consider that this difficulty in determining the boundaries of fast and slow streams contributes to the large values of $d V_{\|}$suggested by fits to IPS observations during the intervals considered in this paper, although the difficulty in distinguishing between variation in the bulk flow speed and random variations in the perpendicular velocity may also play a role (Canals et al., 2002). We have attempted to minimise the effects of these uncertainties, but improvements in the IPS analysis method are probably required to obtain more certain estimates of the variation in flow speed over different regions of the ray path (as discussed in the next section).

Once a satisfactory fit had been obtained, the IPS results were then mapped ballistically to a constant distance of $30 R_{S}$ (in all cases the velocity used for mapping the observations to $2.5 R_{S}$ and then to $30 R_{S}$ was that obtained by fitting the data, as discussed in Sect. 2). When possible (as during CR 1976), in situ data was been mapped inwards to $30 R_{S}$ using a hybrid MHD/ballistic model (Riley et al., 2001), in which MHD mapping is used to trace streams of solar wind in to $75 R_{S}$ and ballistic mapping used to trace flow from $75 R_{S}$ to $30 R_{S}$. When this model could not be used (CR 1953, 1963, 1967), ballistic mapping employing the velocities observed in situ was used to trace the observations back to $30 R_{S}$.

The most serious problems introduced by ballistic mapping occur when attempting to trace measurements made at large heliocentric distances back towards the Sun. As streams of solar wind with different velocities flow out from the Sun, they interact, with faster flow carried under slower flow from higher Carrington longitudes (e.g. Sarabhai, 1963; Gosling et al., 1972; Burlaga, 1974; Pizzo, 1989). This leads to the formation of a compression region on the leading edge of the faster stream and a rarefaction region on the trailing edge, both with velocities intermediate between those of the original streams. As the interaction region develops, narrow streams may become entirely subsumed within it, losing their original discrete character. These regions of interaction - both between fast and slow streams and (we suggest) between regions of slow flow with different velocities - are well developed by $1 \mathrm{AU}$, so that the intermediate velocities generated in them are "folded into" the velocities measured in situ and by IPS observations at larger distances from the Sun (Breen et al., 1998). Mapping these data back ballistically treating each packet of fluid as an individual free-flying element which does not interact with its neighbours - therefore, ignores this history of interaction and will lead to flow from the boundaries between regions of flow, with different velocities being traced to the wrong longitudes. This effect is apparent in the ballistically-mapped spacecraft data, where flow from the boundary between fast and slow streams appears to move "backwards" in longitude (e.g. in the ballisticallymapped Ulysses data from CR 1976 near $50^{\circ}-60^{\circ}$ longitude). The inaccuracies introduced in the mapping process can be significantly reduced by employing a MHD model, since models of this type do not permit adjacent streams to interpenetrate. The improvement in fit between the IPS results and the Ulysses measurements from CR 1976, when the latter were mapped to $30 R_{S}$ using the Riley et al. (2001) model, can be clearly seen from a comparison of Figs. $6 \mathrm{c}$ and $\mathrm{d}$. 
The MHD-mapped Ulysses results shown in Fig. 6d, nevertheless, show rather less variation in velocity than the IPS data. This does not appear to be due to IPS observations from inside the acceleration region of the slow wind underestimating its final flow speed. With the exception of the slow stream detected in the observation of $0431+206$ on 23 May 2001 at $30 R_{S}$, all observations at distances inside $33 R_{S}$ suggested fast flow. In spite of this, the smaller variation in velocities at Ulysses should perhaps not be unexpected, since differences in velocity between adjacent streams should be suppressed by interaction as the distance from the Sun increases and the angle of the Parker spiral steepens (e.g. Pizzo and Gosling, 1994). The Ulysses data will always have these effects of prior interaction "folded into" it and might, therefore, be expected to show a smaller variation in velocity than seen closer to the Sun. The MHD method does, however, minimise systematic errors in the longitudes that streams are mapped to.

Hoeksema (private communication, 2001) suggested that a more physically correct methodology would be to map the IPS observations out to the distances of the in situ measurements. This would mean that the mapping would start with a set of observations in which the effects of prior interaction would be considerably smaller than in the in situ data, and subsequent interaction out to $215 R_{S}$ and beyond would be easy to detect as the ray paths for regions with different outflow speeds drew together or moved away from each other. We consider that this approach has considerable potential for improving our understanding of the evolution of velocity structures in the solar wind. Preliminary studies to test the method using a ballistic mapping appear encouraging (Canals, 2002) and we intend to extend this approach using MHD modelling in a forthcoming paper.

The large variations in velocity (the large values of $d V_{\|}$) suggested by some IPS observations may arise from uncertainty concerning the position of boundaries between regions of fast and slow flow (e.g. Breen et al., 2000a) or to difficulty in distinguishing between the effects of random perpendicular velocities and variations in bulk outflow speed (Canals et al., 2002), although this latter effect should only be significant for slow wind observations during the passage of CMEs. Improvements to the methods used to generate the whitelight maps by adopting the approach described by Wang et al. (1997) could greatly reduce the uncertainties in the position of stream boundaries, while improvements to IPS fitting methods - perhaps using the parameter-search methods developed for forward modelling (e.g. Charbonneau, 1995) could significantly reduce the confusion between random perpendicular velocities and variations in bulk speed.

\section{Conclusions and future developments}

The results from periods when EISCAT IPS observations and in situ measurements were sampling solar wind from similar latitudes and longitudes and when the measurements were unaffected by transients (such as the CME seen by Wind during CR 1967) showed good agreement, with the longi- tudinal variation in flow speed seen in IPS results and in situ measurements being very similar. This good agreement is particularly apparent in the Wind/IPS results from CR 1953, 1963 and 1976, when IPS measurements from within $\pm 10^{\circ}$ latitude of the position of Wind were compared with the corresponding in situ data (Figs. 5a, b, and d) and in the comparison with ecliptic-pass Ulysses data from CR 1967 (Fig. 6c and d). There was a poor match between IPS speeds and those measured in situ by Ulysses during CR 1963, which is consistent with the EISCAT measurements, at latitudes slightly south of those made by Ulysses, sampling outflow from an equatorwards extension of the southern polar hole. Ulysses had not reached these latitudes and so it did not observe this fast flow. The poor agreement between Wind measurements and IPS observations during CR 1967 is best explained by showing an Earth-directed CME passing over Wind - this CME was not detected by EISCAT since there were no observations at the right time to catch it moving out across the ray path. These two periods of poor agreement are thus easily understood, but the results of a comparison between IPS and Ulysses data during CR 1953 are, perhaps, more interesting. During this period the longitudinal variation in IPS and in situ speeds was similar, with solar wind speed increasing from $270^{\circ}$ to $340^{\circ}$ longitude, but the mean speed seen in the IPS results was significantly lower than that measured in situ, while the degree of variation in the IPS speeds (both in terms of the longitudinal variation of the bulk speeds and in the spread in velocities suggested by $\left.d V_{\|}\right)$ was significantly greater than in the in situ results (Fig. 6a). Although this was the most striking example, all observations of regions where strong longitudinal gradients in solar wind speed existed showed a larger variation in velocity in IPS measurements than in situ results, with faster "fast" flow in IPS data and slower "slow" flow.

The mean IPS velocities were often lower than those measured in situ in these regions. Some of the slowest speeds observed by IPS do come from within the slow wind acceleration region, but this is by no means always the case (e.g. the flow speed of $264 \pm 124 \mathrm{~km} / \mathrm{s}$ observed with a P-point distance of $43 R_{S}$ on 20 May 2000), while the higher fast wind flow speeds seen in IPS data cannot be explained in terms of the solar wind acceleration profile. It is quite possible that the IPS measurements underestimate the extent of the faster streams, as regions of fast flow contribute less scintillation than slow streams (e.g. Coles, 1996; Fallows et al., 2002b) and so they are difficult to resolve if they do not lie close to the P-point. Furthermore, as interaction regions develop, the compression regions at their leading edge increase in extent and density, and may come to dominate the IPS observation (Breen et al., 1998). Since the compression regions will have velocities intermediate between those of the initial faster and slower streams, the effect will be for the IPS observations to be biased towards detecting slow and intermediate velocity features. The inner regions of the solar wind may, therefore, be even more variable in velocity than suggested by the EISCAT results presented here. More detailed modelling of the evolution of solar wind streams - probably using MHD mod- 
elling - will be required to take full account of these effects.

We have considered the variation of the differences between the speeds measured by IPS and in situ, and by similar latitudes and over the same range of longitudes (IPS observations from $\pm 10^{\circ}$ latitude of the in situ measurements, in situ measurement averaged over $\pm 20^{\circ}$ of the longitude of the IPS P-point or the width of the IPS stream, whichever was less) in terms of the variation of in situ velocity over the same longitude range, to test whether there is indeed a clear relationship between longitudinal gradients in velocity and changes in velocity between the IPS and in situ measurements. Figure 8 shows the difference between the velocity observed by IPS and in situ divided by the value of $d V_{\|}$required to fit the IPS data and plotted against the standard deviation of in situ speeds over the longitude range corresponding to each IPS data point. There is a suggestion that the difference between IPS and in situ speeds increases when there are significant longitudinal variations in the in situ speed, but the degree of correlation is low.

The success of the MHD model in mapping data from in situ measurements at $300 R_{S}$ to $30 R_{S}$ during CR 1976 is important, as we hope to use this model to extend our study closer into the Sun and compare the longitudinal variation of in situ and IPS wind speeds with the outflow velocities measured in the corona by UVCS. The IPS and in situ measurements during the Ulysses ecliptic pass were closely coordinated with UVCS observations covering heliocentric distances of 2-6 $R_{S}$ above the west limb of the Sun and with LASCO measurements. We hope to use the SAIC MHD model to relate these data sets and determine the regions of origin of streams with different velocities during this interval.

A possible interpretation of the results presented in this paper is that narrow fast and slow streams are interacting to produce a solar wind at the Earth's orbit which is less variable in velocity than that near the Sun, with discrete fast streams subsumed into growing interaction regions with intermediate velocities. This effect would become more pronounced as the distance from the Sun increases, which is consistent with the Ulysses/IPS comparisons from CR 1953, when Ulysses lay at almost $1000 R_{S}$ - the greatest heliocentric distance of any of the observations used in this study. The results strongly suggest that there is significant evolution taking place with distance in the solar maximum solar wind, with interaction between narrow, fast streams and flanking, slow streams, producing a wind that is less variable in velocity, although faster, on average, than the original slow wind. This, in turn, would suggest that the maximum flow speeds seen in situ at $1 \mathrm{AU}$ and by Ulysses may be significantly slower than the fastest wind at the outer edge of the acceleration region. It would also be consistent with the results of 3-D simulations of the development of co-rotating interaction regions (Pizzo and Gosling, 1994) which show substantial blurring of the fast stream between $1 \mathrm{AU}$ and $5 \mathrm{AU}$.

The results reported in this paper demonstrate that it is possible to make quantitative comparisons of IPS and in situ measurements of specific streams of solar wind under the highly-variable conditions prevailing at solar maximum. The

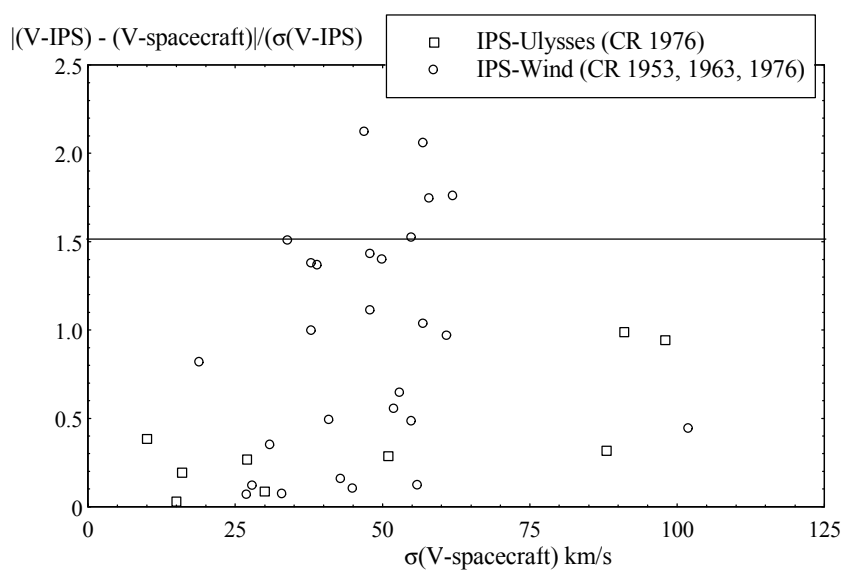

Fig. 8. Variation of $\left|V_{\text {IPS }}-V_{\text {in situ }}\right| / d V_{\|}($IPS $)$with standard deviation within in situ speed $\left(\sigma V_{\text {in situ }}\right)$ over the range of longitudes occupied by the central $40^{\circ}$ of the IPS ray path. There is a suggestion that the largest discrepancies between the IPS and in situ speeds occur when $V_{\text {in situ }}$ is large - that is, when steep longitudinal gradients in velocity exist. The degree of correlation, however, is low ( $18 \%$ for Wind and IPS data). IPS observations which may lie in strong scattering or within the slow wind acceleration region have been omitted, as have observations which may be dominated by the passage of a CME.

task proved considerably more complex than we had initially anticipated - a common experience in studies of the solar wind at solar maximum - but has led to an improved understanding of the problems of relating these widely-separated measurements.

The agreement between the IPS results and the in situ measurements improved significantly when it was possible to use the SAIC MHD mapping routines, to project the in situ measurements back towards the Sun, suggesting that some of the disagreement between the data sets during the earlier intervals considered might have been due to the shortcomings of the ballistic mapping method. We intend to develop this work using MHD modelling to trace IPS observations out to in situ distances (as discussed in Sect. 4) and by introducing additional data sets in the form of UVCS measurements of velocities in the corona and measurements of solar wind speeds at distances of 40-200 $R_{S}$ from the $327 \mathrm{MHz}$ Toyokawa IPS system (Kojima and Kakinuma, 1990), which makes considerably more observations per day than are possible with EISCAT, although it cannot observe so close to the Sun (Moran et al., 2000). We hope to present the results of this extended study in a future paper.

Acknowledgements. We would like to thank the director and staff of EISCAT for the use of the EISCAT IPS data used in this study. EISCAT is supported by the scientific research councils of Finland, France, Germany, Japan, Norway, Sweden and the UK. Particular thanks are due to G. Wannberg, A. Westmann and J. Markkanen for making the May 2001 observations possible and to A. P. Van Eyken for assistance in making EISCAT available for intensive IPS observations. The IPS analysis routines used in this study were developed at University of California, San Diego and we would like 
to thank W. A. Coles for making them available to us. The Ulysses SWOOPS data are used courtesy of D. J. McComas. The Wind SWE data are used courtesy of K. W. Ogilvie, principal investigator. This work (at M.I.T.) is supported in part by NASA. Two of us (ARB - PPARC AF, RAF) were supported by PPARC during the period when this work was carried out. AC was supported by the University of Wales, Aberystwyth. The work carried out by PR was supported by the NASA Sun-Earth Interaction Theory Program.

Topical Editor M. Lester thanks R. Forsyth and another referee for their help in evaluating this paper.

\section{References}

Armstrong, J. and Coles, W. A.: Analysis of three-station interplanetary scintillation data, J. Geophys. Res., 77, 4602-4610, 1972.

Bame, S. J., McComas, D. J., Barraclough, B. L., Phillips, J. L., Sofaly, K. J., Chavez, J. C., Goldstein, B. E., and Sakurai, R. K.: The Ulysses solar wind plasma experiment, Astron. Astrophys. Suppl., 922, 237, 1992.

Behannon, K. W., Burlaga, L. F., and Hewish, A.: Structure and evolution of compound streams at less than or equal to $1 \mathrm{AU}, \mathrm{J}$. Geophys. Res., 96, 21 213-21 225, 1991.

Bothmer, V. and Schwenn, R.: The structure and origin of magnetic clouds in the solar wind, Ann. Geophysicae, 16, 1-24, 1998.

Bourgois, G., Coles, W. A., Daigne, G., Silen, J., Turunen, T., and Williams, P. J. S.: Measurements of the solar wind speed with EISCAT, Astron. Astrophys., 144, 452-462, 1985.

Breen, A. R., Coles, W. A., Grall, R. R., Klinglesmith, M. T., Markkanen, J., Moran, P. J., Tegid, B., and Williams, P. J. S.: EISCAT measurements of the solar wind, Ann. Geophysicae, 14, 1235-1245, 1996.

Breen, A. R., Moran, P. J., Varley, C. A., Wilkinson, W. P., Williams, P. J. S., Coles, W. A., Lecinski, A., and Markkanen, J.: Interplanetary scintillation observations of interaction regions in the solar wind, Ann. Geophysicae, 16, 1265-1282, 1998.

Breen, A. R., Mikic, Z., Linker, J. A., Lazarus, A. J., Thompson, B. J., Moran, P. J., Varley, C. A., Williams, P. J. S., Biesecker, D. A., and Lecinski, A.: Interplanetary scintillation measurements of the solar wind during Whole Sun Month: linking coronal and in situ observations, J. Geophys. Res., 104, 9847-9870, 1999.

Breen, A. R., Moran, P. J., Varley, C. A., Williams, P. J. S., Lecinski, A., Thompson, B. J., and Harra-Murnion, L.: Interplanetary scintillation measurements of the solar wind above low-latitude coronal holes, Adv. Space. Res., 26(5), 790-792, 2000a.

Breen, A. R., de Forest, C. F., Thompson, B. J., McKenzie, J. F., Modigliani, A., Moran, P. J., Varley, C. A., and Williams, P. J. S.: Comparisons of interplanetary scintillation and optical measurements of solar wind acceleration with model results, Adv. Space Res., 26(5), 781-784, 2000b.

Breen, A. R., Tappin, S. J., Jordan, C. A., Thomasson, P., Moran, P. J., Fallows, R. A., Canals, A., and Williams, P. J. S.: Simultaneous interplanetary scintillation and optical measurements of the acceleration of the slow solar wind, Ann. Geophysicae, 18, 995-1002, 2000c.

Breen, A. R., Thompson, B. J., Kojima, M., Biesecker, D. A., Canals, A., Fallows, R. A., Linker, J. A., Lazarus, A. J., Mikic, Z., Moran, P. J., and Williams, P. J. S.: Measurements of the Solar Wind over a wide range of heliocentric distances - a comparison of results from the first three Whole Sun Months, J. Atmos. Sol.-Terr. Phys., 62, 1527-1544, 2000d.

Breen, A. R., Canals, A., Fallows, R. A., Moran, P. J., and Kojima, M.: Large-scale structure of the solar wind from interplanetary scintillation measurements during the rising phase of cycle 23, Adv. Space Res., 29(3), 379-388, 2002a.

Breen, A. R., Thomasson, P., Jordan, C. A., Tappin, S. J., Fallows, R. A., Canals, A., and Moran, P. J.: Interplanetary scintillation and optical measurements of slow and fast solar wind acceleration near solar maximum, Adv. Space Res., in press, 2002b.

Brueckner, G. E., Howard, R. A., Koomen, M. J., Korendyke, C. M., Michels, D. J., Moses, J. D., Socker, D. G., Dere, K. P., Lamy, P. L., Llebaria, A., Bout, M. V., Schwenn, R., Simnett, G. M., Bedford, D. K., and Eyles, C. J.: The large-angle spectroscopic coronagraph (LASCO), Sol. Phys., 162, 357-402, 1995.

Burlaga, L. F.: Interplanetary stream interfaces, J. Geophys. Res., 79, 3717-3725, 1974.

Canals, A.: Interplanetary scintillation studies of the solar wind near solar maximum, Ph.D. thesis, to be submitted to the University of Wales, April, 2002.

Canals, A., Breen, A. R., Ofman, L., Moran, P. J., and Fallows, R. A.: Estimating random transverse velocities in the fast solar wind from EISCAT Interplanetary scintillation measurements, Ann. Geophysicae, (this issue), 2002.

Charbonneau, P.: Genetic algorithms in astronomy and astrophysics, Astrophys. J.(supp.), 101, 309-334, 1995.

Coles, W. A., Esser, R., Løvhaug, U.-P., and Markkanen, J.: Comparison of solar wind velocity measurements with a theoretical acceleration model, J. Geophys. Res., 96, 13 849-13 859, 1991.

Coles, W. A.: Interplanetary scintillation observations of the highlatitude solar wind, Space. Sci. Rev., 72, 211-222, 1995.

Coles, W. A.: A bimodal model of the solar wind, Astrophys. Space Sci., 243, 87-96, 1996.

Dennison, P. A. and Hewish, A.: The solar wind outside the plane of the ecliptic, Nature, 213, 343-346, 1967.

Fallows, R. A.: Studies of the solar wind throughout a solar cycle, Ph.D. Thesis, University of Wales, Aberystwyth, 2001.

Fallows, R. A., Breen, A. R., Moran, P. J., Canals, A., and Williams, P. J. S.: The high-latitude solar wind in EISCAT IPS data, 19911999, Adv. Space Res., in press, 2002a.

Fallows, R. A., Williams, P. J. S., and Breen, A. R.: EISCAT measurements of solar wind speed and the associated level of interplanetary scintillation, Ann. Geophysicae, (this issue), 2002b.

Gazis, P. R.: Synoptic maps of solar wind parameters from in situ spacecraft observations, J. Geophys. Res., 100, 3383-3393, 1995.

Gosling, J. T., Hundhausen, A. J., Pizzo, V., and Asbridge, J. R.: Compressions and rarefactions in the solar wind: Vela 3, J. Geophys. Res., 77, 5442-5454, 1972.

Grall, R. R.: Remote sensing observations of the solar wind near the sun, Ph.D. Thesis, University of California, San Diego, 1995.

Grall, R. R., Coles, W. A., Klinglesmith, M. T., Breen, . R., Williams, P. J. S., and Esser, R.: Rapid acceleration of the polar solar wind, Nature, 1996.

Hewish, A., Scott, P. F., and Willis, D.: Interplanetary scintillation of small-diameter radio sources, Nature, 203, 1214-1217, 1964.

Hyder, C. L. and Lites, B.W.: Ha Doppler brightening and Lymana Doppler dimming in moving Ha prominences, Sol. Phys., 14, 147-158, 1970.

Klinglesmith, M. T.: The polar solar wind from 2.5 to 40 solar radii: results of intensity scintillation measurements, $\mathrm{Ph} . \mathrm{D}$. thesis, University of California, San Diego, 1997.

Kohl, J. L., Esser, R., Gardner, L. D., Habbal, S., Daigneau, P. S., Dennis, E. F., Nystrom, G. U., Panasyuk, A., Raymond, J. C., Smith, P. L., Strachan, L., van Ballegooijen, A. A., Noci, G., Fineschi, S., Romoli, M., Ciaravella, A., Modigliani, A., Huber, 
M. C. E., Antonucci, E., Benna, C., Giordano, S., Tondello, G., Nicolosi, P., Naletto, G., Pernechele, C., Spadaro, D., Poletto, G., Livi, S., Von der Luhe, O., Geiss, J., Timothy, J. G., Gloeckler, G., Allegra, A., Basile, G., Brusa, R., Wood, B., Seigmund, O. H. W., Fowler, W., Fisher, R., and Jhabvala, M.: The ultraviolet coronagraph spectrometer for the solar and heliospheric observatory, Sol. Phys., 162, 213-356, 1995.

Kojima M. and Kakinuma, T.: Solar-cycle dependence of global distribution of solar wind speed, Space Sci. Rev., 53, 173-222, 1990.

Kojima, M., Tokumaru, M., Watanabe, H., Yokobe, A., Asai, K., Jackson, B. V., and Hick, P. L.: Heliospheric tomography using interplanetary scintillation observations -2 : Latitude and heliocentric distance dependence of solar wind structure at $0.1-1$ AU, J. Geophys. Res., 103, 1981-1989, 1998.

Krieger, A. S. and Timothy, A. F.: A coronal hole and its identification as the source of a high velocity solar wind stream, Sol. Phys., 29, 505-525, 1973.

Leinart, C. and Jackson, B. V.: The global solar wind changes over cycle 21: A combination of Helios photometer, in situ and interplanetary scintillation data, Astrophys. J., 505, 984-992, 1998.

Linker, J. A., Mikic, Z., Biesecker, D. A., Forysth, R. J., Gibson, S. E., Lazarus, A. J., Lecinski, A. A., Riley, P., Szarbo, A., and Thompson, B. J.: Magnetohydrodynamic modelling of the solar corona during Whole Sun Month, J. Geophys. Res., 104, 98099830, 1999.

McComas, D. J., Barraclough, B. L., Funsten, H. O., Gosling, J. T., Santiago-Munoz, E., Skoug, R. M., Goldstein, B. E., Neugebauer, M., Riley, P., and Balogh, A.: Solar wind observations over Ulysses' first polar orbit, J. Geophys. Res., 105(A5), 10 419-10 433, 2000.

Massey, W.: Measuring Intensity Scintillations at the Very Long Baseline Array (VLBA) to probe the solar wind near the Sun, M.Phil. Thesis. University of California, San Diego, 1998.

Mikic, Z. and Linker, J. A.: The large-scale structure of the solar corona and inner heliosphere, in Solar Wind Eight, (Eds) Winterhalter, D., et al., A.I.P. Conf. Proc., College Park, Md., 382, pp. 104, 1996.

Miyake, W., Kobayashi, K., Oyama, K. I., Mukai, T., Abe, T., Teresawa, T., Saito, T., Hirao, K., Lazarus, A. J., and Johnstone, A. D.: Multi-spacecraft observations of heliographic structure of the solar wind speed, Planet. Space Sci., 36, 1329-1342, 1988.

Moran, P. J., Breen, A. R., Canals, A., Markkanen, J., Janardhan, P., Tokumaru, M., and Williams, P. J. S.: Observations of interplanetary scintillation during the 1998 whole sun month: a comparison between EISCAT, ORT and Nagoya data, Ann. Geophysicae, 18, 1003-1008, 2000.

Neupert, W. M. and Pizzo, V.: Solar coronal holes as sources of recurrent geomagnetic disturbances, J. Geophys. Res., 79, 37013709, 1974.

Nolte, J. T., Krieger, A. S., Timothy, A. F., Gold, R. E., Roelof, E. C., Vaiana, G., Lazarus, A. J., Sullivan, J. D., and McIntosh, P. S.: Coronal holes as sources of solar wind, Sol. Phys., 46, 303-322, 1976.

Ogilvie, K. W., Chorney, D. J., Fitzenreiter, R. J., Hunsaker, F., Keller, J., Lobell, J., Miller, G., Scudder, J. D., Sittler, Jr., E. C., Torbert, R. B., Bodet, D., Needell, G., Lazarus, A. J., Steinberg, J. T., Tappan, J. H., Mavretic, A., and Gergin, E.: SWE, a comprehensive plasma instrument for the Wind spacecraft, Space Sci. Rev, 71, 55-77, 1995.

Phillips, J. L., Balogh, A., Bame, S. J., Goldstein, B. E., Gosling, J. T., Hoeksema, J. T., McComas, D. J., Neugebauer, M., Shee- ley, N. R., and Yang, Y. M.: Ulysses at 50 south: Constant immersion in the high speed solar wind, Geophys. Res. Lett., 12, 1105-1108, 1994.

Pizzo, V.: 2-D corotating stream fronts near the ecliptic plane in the inner solar wind, J. Geophys. Res., 94, 8673-8684, 1989.

Pizzo, V. J. and Gosling, J. T.: 3-D simulation of high-latitude interaction regions: comparison with Ulysses results, Geophys. Res. Lett., 21, 2063-2066, 1994.

Rao, A., Pramesh, S., Ananthakrishnan, V., Balasubramanian, V., and Coles, W. A.: Very long baseline IPS observations of the solar wind speed in the fast polar streams, Proceedings of International Solar Wind 8 Conference, p. 94, 1995.

Rhodes, E. J. and Smith, E. J.: Multi-spacecraft observations of the heliographic latitude-longitude structure in the solar wind, J. Geophys. Res., 86, 8877-8892, 1981.

Rickett, B. J. and Coles, W. A.: Evolution of the solar wind structure over a solar cycle: Interplanetary scintillation speed measurements compared with coronal observations, J. Geophys. Res., 96,(A2), 1717-1736, 1991.

Riley, P., Gosling, J. T., McComas, D. J., Pizzo, V. J., Luhmann, J. G., Biesecker, D. A., Forsyth, R. J., Hoeksema, J. T., Lecinski, A., and Thompson, B. J.: Relationship between Ulysses plasma observations and solar observations during the Whole Sun Month campaign, J. Geophys. Res., 104, 9871-9879, 1999.

Riley, P., Linker, J. A., and Mikic, Z.: An empirically-driven global MHD model of the solar corona and inner heliosphere, J. Geophys. Res., 106, 15 889-15 901, 2001.

Rishbeth, H. and Williams, P. J. S.: Ionospheric Radar: the system and its early results, Mon. Not. R. Astr. Soc., 26, 478-512, 1985.

Sarabhai, V.: Some consequences of nonuniformity of solar wind velocity, J. Geophys. Res., 68, 1555-1557, 1963.

Schwenn, R.: Large-Scale structures of the interplanetary medium, in: Physics of the inner heliosphere 1, (Eds) Schwenn, R. and Marsch, E., pub. Springer, Berlin Heidleberg, New York, pp. 99$181,1990$.

Sheeley, N. R., Wang, Y. M., Hawley, S. H., Brueckner, G. E., Dere, K. P., Howard, R. A., Koomen, M. J., Korendyke, C. M., Michels, D. J., Paswaters, S. E., Socker, D. G., St. Cyr. O. C., Wang, D., Lamy, P. L., Llebaria, A., Schwenn, R., Simnett, G. M., Plunkett, S., and Biesecker, D. A.: Measurements of flow speeds in the corona between 2 and 30 R-circle dot, Astrophys. J., 484, 472-478, 1997.

Snyder, C. W. and Neugebauer, M.: The relation of Mariner 2 plasma data to solar phenomena, in: The Solar Wind, (Eds) Mackin, R. J. and Neugebauer, M., Pergamon Press, New York, pp. 25-34, 1966.

Spangler, S. R. and Sakurai, T.: Radio interferometer observations of solar wind parameters from the orbit of Helios to the solar corona, Astrophys. J., 445, 999-1016, 1995.

Tappin, S. J., Simnett, G. M., and Lyons, M. A.: A determination of the outflow speeds in the lower solar wind, Astron. Astrophys., 350, 302-309, 1999.

Wang, Y.-M., Sheeley, N. R., Howard, R. A., Kraemer, J. R., Rich, N. B., Andrews, M. D., Brueckner, G. E., Dere, K. P., Koomen, M. J., Korendyke, C. M., Michels, D. J., Moses, J. D., et al. : Origin and evolution of coronal streamer structure during the 1996 minimum activity phase, Astrophys. J., 485, 875-889, 1997.

Woch, J., Axford, W. I., Mall, U., Wilken, B., Livi, S., Geiss, J., Gloeckler, G., and Forsyth, R. J.: SWICS/Ulysses observations: The three dimensional heliosphere in the declining/minimum phase of the solar cycle, Geophys. Res. Lett., 24, 2885-2888, 1997. 